\title{
Fractional-order model representations of apparent vascular compliance as an alternative in the analysis of arterial stiffness: An in-silico study
}

\author{
Mohamed A. Bahloul and Taous-Meriem Laleg Kirati \\ Computer, Electrical and Mathematical Sciences and Engineering Division, \\ King Abdullah University of Science and Technology, Thuwal, Makkah 23955-6900, Saudi Arabia \\ E-mail: \{mohamad.bahloul@kaust.edu.sa; taousmeriem.laleg@kaust.edu.sa\}.
}

\begin{abstract}
Recent studies have demonstrated the advantages of fractional-order calculus tools for probing the viscoelastic properties of collagenous tissue, characterizing the arterial blood flow and red cell membrane mechanics, and modeling the aortic valve cusp. In this article, we present novel lumpedparameter equivalent circuit models of the apparent arterial compliance using a fractional-order capacitor (FOC). FOC, which generalizes capacitors and resistors, displays a fractionalorder behavior that can capture both elastic and viscous properties through a power-law formulation. The proposed framework describes the dynamic relationship between the blood pressure input and blood volume, using linear fractionalorder differential equations. The results show that the proposed models present reasonable fit performance with in-silico data of more than 4,000 subjects. Additionally, strong correlations have been identified between the fractional-order parameter estimates and the central hemodynamic determinants as well as pulse wave velocity indexes. Therefore, fractional-order based paradigm of arterial compliance shows prominent potential as an alternative tool in the analysis of arterial stiffness.

Keywords-Cardiovascular system, Apparent compliance, Input Impedance, Fractional order capacitor, Arterial stiffness
\end{abstract}

\section{INTRODUCTION}

$\mathbf{O}$ VER the last decades, arterial models have been proven to be extremely useful and effective in unraveling cardiovascular diseases [1], in the medical intervention planning [2], in diseases' treatment and monitoring [3], and in the design and testing of medical devices and simulators [4], [5]. Besides, arterial models have shown great potential in the noninvasive evaluation of physiological parameters, which are not directly accessible, such as the arterial compliance and stiffness [6], [7], [8].

Vascular compliance is defined as the ability of a particular arterial vessel to store blood. It describes the capacitance of the vascular wall to dynamically distend and increase the vessel volume with an increase in the transmural pressure or the tendency of the vascular wall to resist and recoil toward its original geometry with compression. In the last few decades, there has been keen interest in understanding the relationship between arterial compliance variation and cardiovascular diseases. The clinical relevance of vascular compliance is due to its fundamental role in pulsatile hemodynamics. Accordingly, several clinical and experimental research studies have been proposed to quantify vascular compliance, and various arterial compliance surrogates have been developed [9]. In this regard, various linear and nonlinear computational methods for the calculation of vascular compliance were presented in the open literature review [10], [11], such as 1) Time decay method, which is known as linear Windkessel model compliance [12], [13], 2) area method, [14], 3) Stroke volume over pulse pressure method, [15], [16], 4) Exponential method and recently 5) compliance-pressure loop approach [17], [18].

Functionally, arterial compliance is demonstrated by the relationship between the stored blood volume's variation and the input blood pressure's variation. Similarly, the concept of total arterial compliance was introduced as the sum of all compliance components of the entire arterial system. Thus, the total compliance describes the global arterial capacity to store blood and is equal to the variation in blood volume in the entire arterial system divided by the systemic input pressure's variation. However, it is known that this ratio is not only governed by the total arterial compliance but also incorporates some other effects such as the pulse wave reflection. Indeed, it is equivalent to the total compliance only at low frequency. Hence, the concept of dynamic arterial compliance-or, equivalently, apparent compliance have been proposed by Quick et al. [19] to show how to estimate the true total compliance correctly from the transfer function relating the blood volume to the input pressure [20] and explain a question of fact as to whether the classical estimation methods of arterial compliance fails to yield to true arterial compliance.

Before the introduction of the "apparent compliance" concept, the transfer function relating blood volume to systemic input pressure is thought to be constant and modeled by a constant capacitance of an ideal capacitor (electrical analog model). This hypothesis is based on the Windkessel concept, which is adopted by the lumpedelement modeling school. The drawbacks of this assumption are reflected in its estimation-based methods of compliance, which doesn't yield to a correct evaluation of the true arterial compliance [21]. Because of the distributed nature of the vascular compliance and resistance within the 
arterial network, the relationship blood Volume/input pressure is frequency-dependent [19]. Accordingly, a time delay between the arterial blood volume and the input pressure occurs. During the past decades, some clinical studies demonstrated the necessity of introducing apparent compliance to extract total compliance. Therefore, a new lumped-parameters modeling framework, which takes into account the complex and frequency-dependence properties of the apparent arterial compliance, have been proposed [22]. These models are based on the idea that the arterial wall is viscoelastic rather than pure elastic. Hence, the Voigt cell model (resistor in series with a capacitor) has been proposed as a suitable candidate to represent the total arterial compliance. The resistor of the Voigt cell displays the viscous losses held by the arterial wall motions, while the capacitor represents the static compliance of the arteries. Through the combination of resistor and capacitor cells gives rise to complex and frequency-dependent compliance, the Voigt model configuration is considered very poor in representing the arterial viscoelasticity properties since it does not account for the stress-relaxation experiment [23]. Therefore, to address this inconsistency, the order of the viscoelastic representation has been increased by adding more viscous and elastic connected elements [22]. The higher-order configuration provided a more accurate but complex configuration, where its complexity is principally due to the enormous number of unknown parameters, which suggests another challenge. Indeed, for higher-order models, the number of parameters to identify is more significant, while the collected real data is small and insufficient. It is also known that reduced-order models are desirable for their simplicity and ease of exploration.

Over the last decades, the fractional-order derivative (FD), defined as a generalization of the standard integer derivative to a non-integer order, has been gaining paramount popularity in modeling and characterizing biological tissues [24], [25]. Because of its non-locality and memory properties, FD has been regarded as a powerful tool for modeling complex physical phenomena that exhibit power-law response or involve memory effects [26], [27]. In recent research, the power-law behavior has been proved in the viscoelasticity characterization of an elastic aorta. The in-vivo and invitro data analysis showed that the FD tools are more convenient to accurately model and describe the arterial wall viscoelastic dynamic response [28], [29], [30], [31], [32]. Besides, a recent study by the authors [33], [34], [35], used fractional-order derivative tools to the wellknown arterial Windkessel paradigm, by replacing the ideal capacitor, which accounts for the total arterial compliance, with a fractional-order capacitor. The preliminary analysis demonstrated that the fractional-order impedance is the right candidate for the accurate assessment of the aortic input impedance. Furthermore, a simple correlation between the main parameters of the central arterial blood pressure and the fractional differentiation operator has been shown. Consequently, the novel fractional-order parameter may have an influential role as a physiological index of the arterial stiffness [36], [37].

This paper introduces and investigates the fractional-order derivative modeling framework for apparent compliance. The proposed modeling framework offers a new paradigm for the physiological interpretation of the frequency-dependent arterial compliance and the interaction between the systemic arterial mechanical properties (viscosity and elasticity). Besides, this study compares the different proposed models as well as with the corresponding integer-order models.

The rest of the paper is organized as follows: in Section II, the preliminaries, the proposed models and the method are presented. Section III shows the results and discussion. Finally, section IV presents the conclusion and future perspectives.

\section{PRELIMINARIES}

\section{A. Input impedance, apparent compliance, and resistance}

Aortic input impedance $\left(Z_{i n}\right)$ and apparent compliance $\left(C_{a p p}\right)$ are considered significant in the characterization of the arterial system, independently of the heart properties. Whereas $Z_{\text {in }}$ describes the ability of the arterial system to hamper the blood flow dynamically, $C_{a p p}$ depicts the capacity of the arterial bed to store blood dynamically. Functionally, $Z_{\text {in }}$ is defined as the dynamic relationship, in the frequency domain, of the arterial blood pressure $\left(P_{i n}\right)$ and blood flow $\left(Q_{i n}\right)$ at the entrance of the systemic circulatory system, that is:

$$
Z_{\text {in }}(\omega)=\frac{P_{\text {in }}(\omega)}{Q_{\text {in }}(\omega)},
$$

where $\omega$ corresponds to the angular frequency. $C_{a p p}$ is defined as the dynamic relationship, in the frequency domain, between the blood volume $V$, and the input aortic blood pressure $\left(P_{\text {in }}\right)$ that is:

$$
C_{a p p}=\frac{V(\omega)}{P_{i n}(\omega)}
$$

Similarly, to the concept of apparent compliance, another frequency dependent transfer function relating $P_{\text {in }}$ to the output blood flow $\left(Q_{\text {out }}\right)$ has been defined as well. It describes the so-called apparent resistance $\left(R_{a p p}\right)$ and it can be formulated as:

$$
R_{\text {app }}=\frac{P_{\text {in }}(\omega)}{Q_{\text {out }}(\omega)}
$$

Based on Quick et al. investigations in [19] and [20], $R_{a p p}$ can be approximated as a constant that is equivalent to the total peripheral resistance (DC component of the input impedance). Additionally, $C_{a p p}$ can be expressed in terms of $Z_{\text {in }}$ and $R_{\text {app }}$ :

$$
C_{a p p}=\frac{R_{a p p}-Z_{i n}}{j \omega R_{a p p} Z_{i n}}
$$


$v(t)$

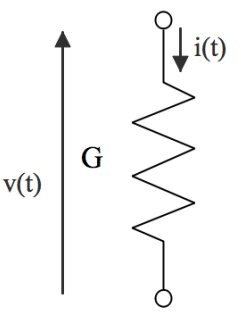

RESISTOR
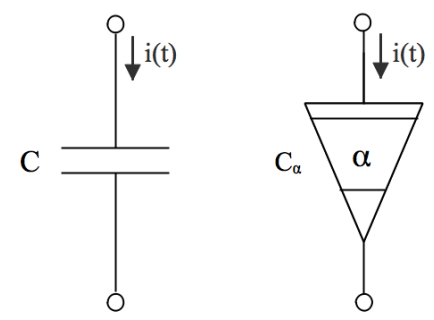

CAPACITOR FRACTIONAL-ORDER CAPACIOTR

$$
\mathrm{i}(\mathrm{t})=\mathrm{G} \cdot \mathrm{D}^{0} \mathrm{v}(\mathrm{t}) \quad \mathrm{i}(\mathrm{t})=\mathrm{C} \cdot \mathrm{D}^{1} \mathrm{v}(\mathrm{t}) \quad \mathrm{i}(\mathrm{t})=\mathrm{C}_{\alpha} \cdot \mathrm{D}^{\alpha} \mathrm{v}(\mathrm{t})
$$

Fig. 1: The schematic diagram for the resistor, capacitor, and fractionalorder capacitor elements along with theirs $i$ - $v$ characteristic relationships. Here, for the fractional-order capacitor, $i(t)=C_{\alpha} D^{\alpha} v(t)$ where $0 \leq \alpha \leq 1$ and $C_{\alpha}$ is the pseudo-capacitance. The bounding values of $\alpha$ represent the discrete conventional elements (Resistor, $C_{\alpha}=G$ when $\alpha=0$; Capacitor, $C_{\alpha}=C$, the capacitance, when $\left.\alpha=1\right)$.

The detailed derivation of (4) is given in the appendix A.

As mentioned in the previous section, the transfer function describing the apparent compliance is frequency-dependent and describes, not only the total arterial compliance but also incorporates other physiological effects such as pulse reflections. At low frequency, $C_{a p p}$ convergences to a value that approximates the true total arterial compliance $\left(C_{t o t}\right)$ :

$$
C_{\text {tot }}=\lim _{w \rightarrow 0} C_{a p p}
$$

\section{B. Fractional-order capacitor (FOC)}

FOC also known as Constant Phase Element [38], is the main building block for developing analog model structure according to FC. FOC is an electrical element that represents a fractional-order derivative relationship between the current, $q(t)$, passing through and the voltage, $p(t)$, across it with respect to time, $t$, as follow:

$$
q(t)=C_{\alpha} D_{t}^{\alpha} p(t)
$$

where $C_{\alpha}$ is a proportionality constant so-called pseudocapacitance, expressed in units of $\left[\right.$ Farad $/$ second $\left.^{1-\alpha}\right]$. The conventional capacitance, $C$, in unit of Farad is related to $C_{\alpha}$ as follow:

$$
C=C_{\alpha} \omega^{\alpha-1} .
$$

The impedance $\left(Z_{F O C}\right)$ of FOC in Laplace domain is given as:

$$
Z_{F O C}(s)=\frac{1}{C_{\alpha} s^{\alpha}} \text {. }
$$

Substituting the Laplace variable, $s$, by $(j \omega)$, (9) can be expressed as:

$$
Z_{F O C}(\omega)=\underbrace{\frac{1}{C_{\alpha}} \omega^{-\alpha} \cos (\phi)}_{G_{r}}-j \underbrace{\frac{1}{C_{\alpha}} \omega^{\alpha} \sin (\phi)}_{H_{r}},
$$

where $\phi$ represents the phase shift given by the formula: $\phi=\alpha \pi / 2$ [rad] or $\phi=90 \alpha$ [degree or $^{\circ}$ ].

As illustrated in Fig. 1 the bounding values of $\alpha$ represent the discrete conventional elements: the resistor when $\alpha=0$ and the capacitor when $\alpha=1$ ). Additionally, from (9), it is clear that as $\alpha$ goes to 0 , the imaginary part $\left(H_{r}\right)$ of $Z_{C}$ vanishes to 0 and hence the FOC characteristic becomes more like that a pure resistor, whereas as $\alpha$ approaches to 1 , the real part $\left(G_{r}\right)$ converges to 0 and hence, FOC operates as a pure capacitors. Furthermore, it has been demonstrated that the characteristics of FOC can be approximated using RC ladder structure [39]. Based on the above properties and in comparison to an integer order model where $\alpha$ is strictly fixed to an integer ( 0 or 1$)$, the parameter $\alpha$ offers extra flexibility for a fractional-order paradigm. In connection with the apparent compliance modeling concept, FOC can be considered as a great candidate that might overcome

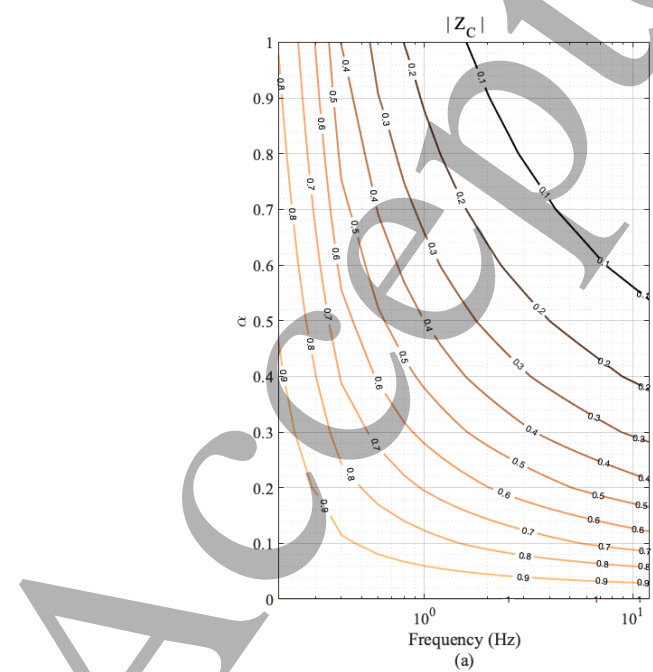

Fig. 2: Modulus of (FOC impedance $Z_{C}$, left side), (the dissipation part $Z_{D}$ ) and (the storage part $Z_{S}$ ) for $C_{\alpha}=1$. Based on the order of the fractional differentiation factor $\alpha$, the modulus, storage and the dissipation parts of the resultant FOC's impedance can have different levels.



ency $(\mathrm{Hz})$

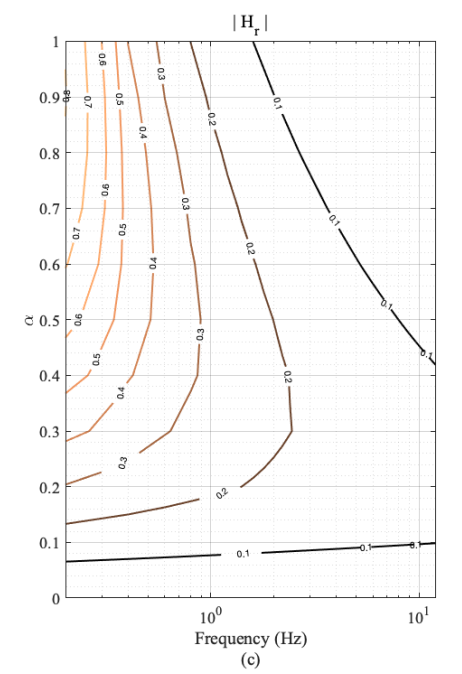




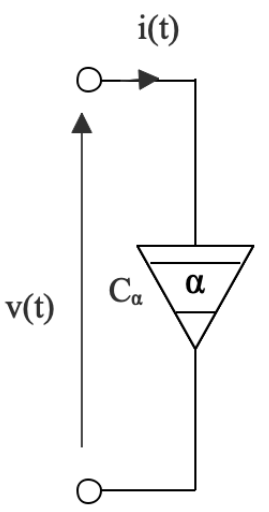

(A) i(t)

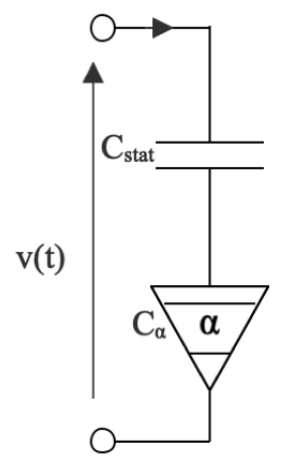

(B) i (t)

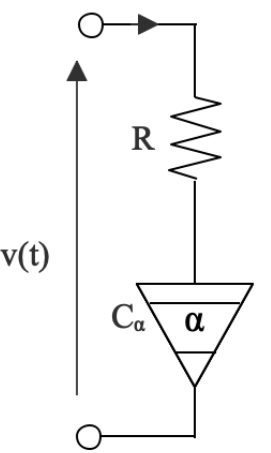

(C)

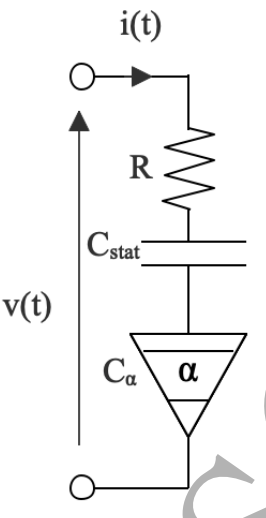

(D)

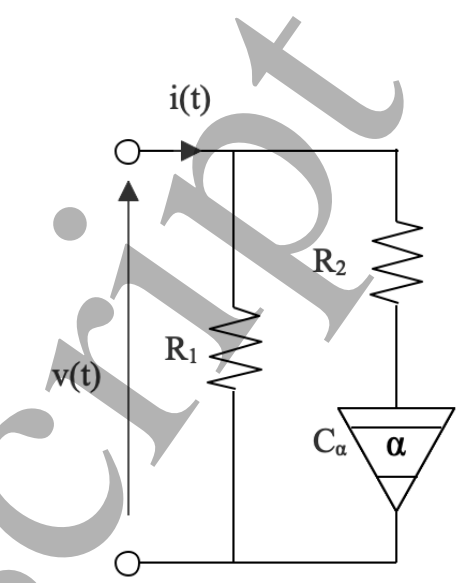

(E)

Fig. 3: Schematic representations of the electrical analog of the proposed fractional-order models Model representation (A): It comprises one fractionalorder capacitor $\left(C_{\alpha}\right)$ that combines both elastic and viscous properties of the arterial system in one single element. Based on the order of the fractional differentiation factor $\alpha$, the elastic and the viscous parts of $C_{\alpha}$ can have different levels. Model representation $(B)$ : It comprises a fractional-order capacitor $\left(C_{\alpha}\right)$ in series with a resistor $\left(C_{\text {stat }}\right)$. $C_{\text {stat }}$ accounts as a static capacitance. Model representation $(C)$ : It comprises a fractional-order capacitor $\left(C_{\alpha}\right)$ in series with a resistor $\left(R_{1}\right) . R_{1}$ accounts as a static resistance. Model representation $(D)$ : It comprises a fractional-order capacitor $\left(C_{\alpha}\right)$ in series with an ideal capacitor $\left(C_{\text {stat }}\right)$ and a resistor $(R) . R_{1}$ accounts as a static resistance and $C_{\text {stat }}$ accounts as a static capacitance. Model representation $(E)$ : It has similar representation as the well-known viscoelastic Maxwell model; however the ideal capacitor was replaced by a fractional-order one $C_{\alpha}$. In all these representations, $C_{\alpha}$ offers a key advantage in characterizing arterial networks. The whole spectrum of dissipative and storage-based viscoelastic mechanisms may be included in a single parameter (the fractional differentiation order).

the discrepancies stemming from integer-order limitation/as follows:

- The proportionality constant $C_{\alpha}$ (pseudo-capacitance) is expressed in unit of $\left[\mathrm{F} \cdot \mathrm{sec}^{1-\alpha}\right]$ that makes, by its very nature, the conventional capacitance $C$, in the unit of [Farad], frequency-dependent, hence FOC has a physical foundation in representing the complex and frequency dependence of $C_{a p p}$.

- Based on the order of the fractional differentiation factor $\alpha$, the storage and the dissipation parts of the resultant FOC's impedance can have different levels, as illustrated in Fig. 2. Thus FOC might offer a key advantage in modeling complex system, that is the whole spectrum of dissipative and storage mechanisms may be included in a single parameter (the fractional differentiation order).

- The equivalent analog circuit of FOC can be viewed as infinity Voigt cells connected in parallel. Hence FOC might lead to a minimal representation of the mechanical properties of the arterial network by using only two parameters $\left(\alpha\right.$ and $\left.C_{\alpha}\right)$.

In biorheological research field, the imaginary, as well as the real part of $Z_{F O C}$, might represent the tissue damping $\left(G_{r}\right)$ and tissue elastance $\left(H_{r}\right)$, respectively:

$$
\left\{\begin{array}{l}
G_{r}(j \omega)=\frac{1}{C_{\alpha} \omega^{\alpha}} \cos \left(\alpha \frac{\pi}{2}\right) \\
H_{r}(j \omega)=-\frac{1}{C_{\alpha} \omega^{\alpha}} \sin \left(\alpha \frac{\pi}{2}\right)
\end{array}\right.
$$

The hysteresivity coefficient $\eta_{r}$ (dimensionless) is defined as:



In general, these parameters are usually used to characterize the heterogeneity of the bio-tissue and are shown to be variable with pathology, as demonstrated for lung tissue for the respiratory system in [40].

\section{MODELS}

\section{A. Fractional order model of the dynamic Volume/Input- pressure relationship}

Recent researches have shown the key advantages of applying fractional calculus tools to describe correctly: 1) the viscoelasticity properties of the collagenous tissues in the arterial bed, 2) analyze the arterial blood flow [31], [32] and red blood cell (RBC) membrane mechanics [30] and, 3) modeling the heart valve cusp [41]. Bearing this in mind, in this part, we introduce the fractionalization of the dynamic relationship of the arterial blood volume and inputpressure. Based on the conservation mass, the arterial blood flow pumped from the heart to the arterial vascular bed $\left(q_{i n}\right)$ can be expressed as:

$$
q_{\text {in }}(t)=q_{\text {stored }}(t)+q_{\text {out }}(t)
$$

where $q_{\text {stored }}$ is the blood stored in the arterial tree, and $q_{\text {out }}$ corresponds to the flow out of the arterial system. As described in (3), qout can be expressed as:

$$
q_{\text {out }}(t)=\frac{1}{R_{\text {app }}} p_{\text {in }}(t) .
$$

Regarding $q_{\text {stored }}$, typically using the conventional definition, it can be determined as the rate of flow by taking the first derivative of the volume equation for the time, whereas, in consideration of the fractional properties of both RBC and the collagenous tissues forming the arterial bed, we allow the differentiation order of the blood volume for time to be real 

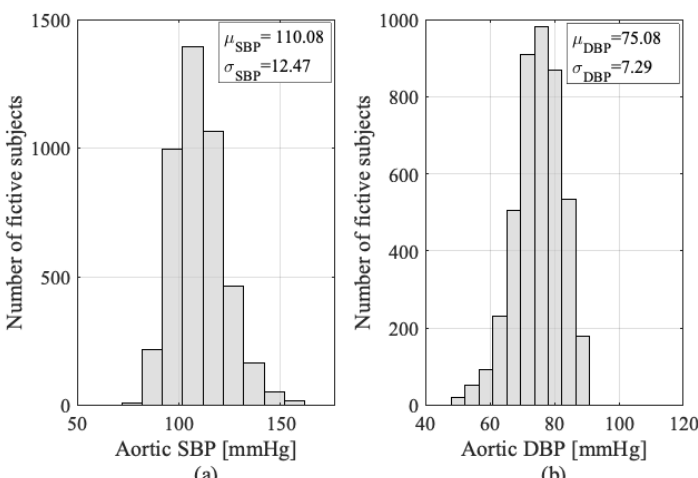

(b)

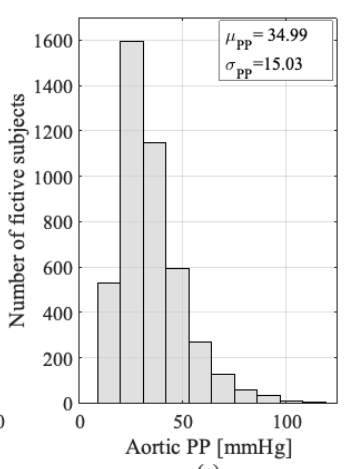

(c)

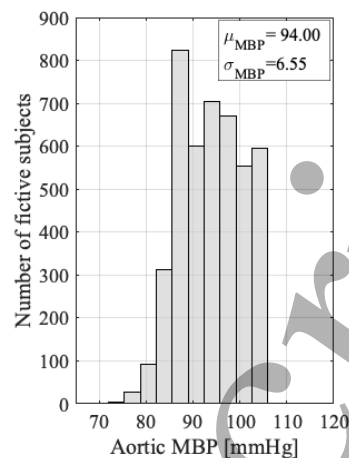

(d)

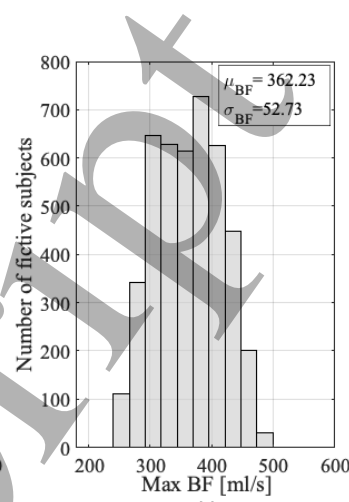

(e)

Fig. 4: Distribution, mean value and standard deviation of: (a) systolic blood pressure (SBP), (b) diastolic blood pressure (DBP), (c) aortic pulse pressure $(A P P=S B P-D B P),(d)$ mean blood pressure $(\mathrm{MBP})$, and (e) the maximum of the blood low (BF) at the level of of ascending aorta for 4,374 virtual subject based in-silico database.

$\left(\alpha \in\left[\begin{array}{ll}0 & 1\end{array}\right]\right)$ and hence applying the fractional-order derivative to this differential equation.

$$
\begin{aligned}
& q_{\text {stored }}(t)=D_{t}^{\alpha} V(t)=\frac{d^{\alpha} V(t)}{d t^{\alpha}}, \\
& q_{\text {stored }}(t)=\underbrace{\frac{d^{\alpha} V(t)}{d^{\alpha} p_{\text {in }}(t)}}_{A_{\alpha}} \frac{d^{\alpha} p_{\text {in }}(t)}{d t^{\alpha}},
\end{aligned}
$$

where $A_{\alpha}$ is a fractional order proportionality constant that can be defined as a fractional order compliance expressed in the unit of $\left[1 / \mathrm{mmHg} \cdot \sec ^{1-\alpha}\right]$. Assuming null initial condition,the Laplace transform of (15) is given as:

$$
Q_{\text {stored }}=A_{\alpha} s^{\alpha} P_{i n} .
$$

The conventional compliance in the unit of $[1 / \mathrm{mmHg}]$ that represents the complex and frequency-dependent apparent compliance can be written as follow:

$$
C_{C}=A_{\alpha} s^{\alpha-1} .
$$

Accordingly, by analogy to the electrical circuit, one may appropriately consider the fractional-order capacitor as a lumped parametric element to stand for the apparent arterial compliance. The voltage is equivalent to the arterial pressure; the electrical charges correspond to the blood volume and the electrical current as of the equivalent of the blood flow.

\section{B. Fractional-order models of the apparent vascular compli-} ance

In this part, we show the derivations of the proposed model based-structures for modeling the apparent arterial compliance. These structures scheme different combinations of FOC along with the conventional resistor and capacitor to display the complex and frequency-dependent behavior of the real dynamic compliance. Fig. 3 shows the proposed electrical analog structures of the proposed models.

1) Model A: It comprises only one single FOC. As detailed in the previous sections, the apparent compliance expressed in unit of $[1 / \mathrm{mmHg}]$ can be written as:

$$
C_{c}^{A}=C_{\alpha} s^{\alpha-1} \text {. }
$$

2) Model B: It comprises an ideal capacitor $\left(C_{\text {stat }}\right)$ accounting for the static compliance and FOC connected in series. The apparent compliance expressed in the unit of $[1 / \mathrm{mmHg}]$ can be written as:

$$
C_{c}^{B}=\frac{C_{\alpha} C_{\text {stat }} s^{\alpha}}{C_{\alpha} s^{\alpha}+C_{s t a t} s}
$$

3) Model C: It comprises a resistor $(R)$ and FOC connected in series. The apparent compliance expressed in the unit of $[1 / \mathrm{mmHg}]$ can be written as:

$$
C_{c}^{C}=\frac{C_{\alpha} s^{\alpha-1}}{1+R C_{\alpha} s} .
$$

4) Model D: It comprises a resistor $(R)$, an ideal capacitor $\left(C_{\text {stat }}\right)$ and FOC connected in series. The apparent compliance expressed in unit of $[1 / \mathrm{mmHg}]$ can be written as:

$$
C_{c}^{D}=\frac{C_{\text {stat }} C_{\alpha} s^{\alpha}}{C_{\text {stat }} s+C_{\alpha} s^{\alpha}+R C_{\alpha} C_{\text {stat }} s^{\alpha+1}} .
$$

5) Model E: It comprises a resistor $\left(R_{1}\right)$ in parallel to a FOC and a resistor $\left(R_{2}\right)$ connected in series. The apparent compliance expressed in unit of $[1 / \mathrm{mmHg}]$ can be written as:

$$
C_{c}^{E}=\frac{1+\left(R_{1}+R_{2}\right) C_{\alpha} s^{\alpha-1}}{R_{1}\left(1+R_{2} C_{\alpha} s^{\alpha}\right)} .
$$

\section{MATERIAL AND METHOD}

A. Pulse wave database for in-silico evaluation of hemodynamics determinants and vascular stiffness indexes

Owing to a lack of real data to validate the proposed approaches, in this study, we utilize a virtual database of simulated pulse waves (PWs) created by Peter H.Charlton et al., [42]. In the following, we provide an overview of the used dataset, and the model explored to generate it. Moreover, we note the principal limitations of this used approach as well as the in-silico dataset.

1) Overview: In this work, we use the publicly available PWs database 1 which is considered a useful resource to evaluate the pre-clinical assessment of PWs analysis algorithms. This database is intended to reproduce a sample of

1'https://peterhcharlton.github.io/pwdb/index.html 
TABLE I: Mean value of systolic blood pressure (SBP), diastolic blood pressure (DBP), aortic pulse pressure $(P P=S B P-D B P)$, mean blood pressure (MBP), and the maximum of the blood low (BF) at the level of of ascending aorta for 4,374 virtual subject based in-silico database.

\begin{tabular}{|c|c|c|c|c|c|c|c|}
\hline Age & Number of subjects & Heart rate & SBP $[\mathrm{mmHg}]$ & DBP $[\mathrm{mmHg}]$ & $\mathrm{MBP}[\mathrm{mmHg}]$ & $\mathrm{APP}[\mathrm{mmHg}]$ & $\mathrm{BF}[\mathrm{ml} / \mathrm{s}]$ \\
\hline \hline \multirow{3}{*}{25} & 243 & 84.11 & $101.07 \pm 7.87$ & $76.67 \pm 5.98$ & $90.75 \pm 6.22$ & $24.3957 \pm 7.09$ & $404.69 \pm 53.87$ \\
\cline { 2 - 8 } & 243 & 72.91 & $99.95 \pm 8.41$ & $74.58 \pm 5.44$ & $89.13 \pm 6.21$ & $25.36 \pm 7.29$ & $389.58 \pm 52.04$ \\
\cline { 2 - 8 } & 243 & 61.72 & $99.04 \pm 8.93$ & $72.54 \pm 4.99$ & $87.43 \pm 6.31$ & $26.49 \pm 7.37$ & $376.65 \pm 50.93$ \\
\hline \hline \multirow{3}{*}{35} & 243 & 87.99 & $104.549 \pm 8.59$ & $78.59 \pm 6.24$ & $93.60 \pm 6.18$ & $25.95 \pm 8.96$ & $394.46 \pm 51.97$ \\
\cline { 2 - 8 } & 243 & 76.80 & $104.36 \pm 8.94$ & $77.33 \pm 5.93$ & $92.78 \pm 6.19$ & $27.02 \pm 9.02$ & $378.43 \pm 50.56$ \\
\hline \multirow{3}{*}{45} & 243 & 65.60 & $104.07 \pm 9.33$ & $75.91 \pm 5.50$ & $91.58 \pm 6.26$ & $28.15 \pm 9.00$ & $363.64 \pm 49.50$ \\
\cline { 2 - 8 } & 243 & 88.93 & $110.01 \pm 9.09$ & $79.53 \pm 6.45$ & $96.86 \pm 6.16$ & $30.47 \pm 10.05$ & $384.97 \pm 50.42$ \\
\hline \multirow{3}{*}{55} & 243 & 77.74 & $110.15 \pm 9.27$ & $78.58 \pm 6.18$ & $96.22 \pm 6.17$ & $31.57 \pm 9.97$ & $368.01 \pm 49.07$ \\
\cline { 2 - 8 } & 243 & 66.54 & $110.18 \pm 9.67$ & $77.39 \pm 5.80$ & $95.24 \pm 6.24$ & $32.78 \pm 10.06$ & $352.91 \pm 48.21$ \\
\cline { 2 - 8 } & 243 & 88.49 & $112.50 \pm 10.35$ & $77.56 \pm 6.64$ & $96.96 \pm 6.12$ & $34.93 \pm 12.37$ & $374.06 \pm 48.72$ \\
\hline \hline \multirow{3}{*}{65} & 243 & 77.30 & $112.88 \pm 10.55$ & $76.76 \pm 6.40$ & $96.42 \pm 6.15$ & $36.11 \pm 12.37$ & $357.28 \pm 47.57$ \\
\cline { 2 - 8 } & 243 & 66.10 & $113.15 \pm 10.94$ & $75.84 \pm 6.05$ & $95.62 \pm 6.22$ & $37.30 \pm 12.47$ & $341.64 \pm 46.86$ \\
\cline { 2 - 8 } & 243 & 76.51 & $116.26 \pm 21.03$ & $74.91 \pm 21.43$ & $96.74 \pm 19.72$ & $42.94 \pm 26.85$ & $345.21 \pm 45.91$ \\
\hline \hline \multirow{3}{*}{75} & 243 & 65.31 & $116.70 \pm 21.20$ & $74.21 \pm 21.36$ & $96.08 \pm 19.78$ & $44.08 \pm 26.82$ & $329.64 \pm 45.47$ \\
\cline { 2 - 8 } & 243 & 85.63 & $117.42 \pm 23.41$ & $71.69 \pm 22.14$ & $95.34 \pm 20.02$ & $49.17 \pm 29.85$ & $341.50 \pm 61.49$ \\
\cline { 2 - 8 } & 243 & 74.43 & $118.10 \pm 14.39$ & $70.02 \pm 8.46$ & $94.52 \pm 6.02$ & $48.07 \pm 19.94$ & $331.84 \pm 44.34$ \\
\hline
\end{tabular}

healthy adults' pulse waves, aged 25-75 years old, in ten-year increments. For each age group, 729 virtual subjects based on pulse waves were created by varying specific cardiac and arterial parameters like the arterial stiffness and heart rate within normal ranges. This database encompasses four arterial PWs: (1) flow velocity, (2) luminal area, (3) pressure and (4) photoplethysmogram pulse waves at different sites of the arterial network such as the ascending aorta, carotid artery, brachial artery, and radial arteries. In this study, PWs at the level of ascending aorta have been investigated to evaluate our approaches. Fig. 4 shows a summary statistic of the aortic blood pressure parameter as well as the maximum blood flow at the level of the ascending aorta, for all virtual subjects. Additionally, we present a detailed statistic summary based on the group age and heart rate in TABLE ?? in the supplementary material. This database presents physiological values with well-balanced distributions.

2) NektarlD, [43], numerical approach for solving $1 D$ equations of blood flow in arterial networks: To generate the database of PWs, Peter H.Charlton et al., used one-dimensional in the time-domain (1D numerical model) known as NektarlD ${ }^{2}$ Nektar1D is an in-house algorithm for solving nonlinear, one-dimensional blood flow equations in a given network of vessels, subject to a specific boundary and initial conditions. Nektar $1 D$ consists of three essential parts:

- Arterial system: In this approach, the arterial network is split into 116 vascular segments, making up the thorax's larger arteries, limbs, and head. Arterial segments were modeled as thin, viscoelastic tubes of constant length and linearly tapered diameter [44].

${ }^{2}$ http://haemod.uk/nektar
- Periodic inflow: A periodic inflow waveform was considered as a boundary condition at the aortic root level, characterizing the pumped left ventricle's blood flow.

- Boundary conditions: The well-known three-element windkessel arterial representation which characterizes the vascular beds was applied as boundary conditions at the outlets of peripheral arterial levels.

In order to generate the investigated data in the present work (samples of healthy adults' pulse waves, aged 25-75 years old, in ten-year increments), the input parameters of the NektarlD were set and varied based on real value and range of cardiovascular properties with respect to age recognized on previous scientific reviews [45], [46], [47], [48], [49], [50], [51], [52], [53], [54]. The input parameters have been varied with age allowing the effects of aging to be examined, and were also varied within normal ranges at each age, permitting the influences of individual parameters to be illustrated[55], [56], [57], [58], [59], [60], [61]. It is worth noting that the adopted approach to generate the insilico database consolidates the connections between some cardiovascular input parameters, including the dependencies of left ventricular ejection time on stroke volume as well as the heart rate (HR) and the arterial stiffness on mean blood pressure and vascular geometry. A complete demonstration of the 1D equations and the numerical scheme used to solve them in NektarlD can be found in the book chapter, [43].

The use of 1D modeling had a great potential to simulate PWs at a range of standard measurement sites and include PW propagation influences of changing arterial properties through the arterial tree that has been demonstrated in pioneering investigations studying the hemodynamic properties [62], [63]. The created database was verified by comparing 


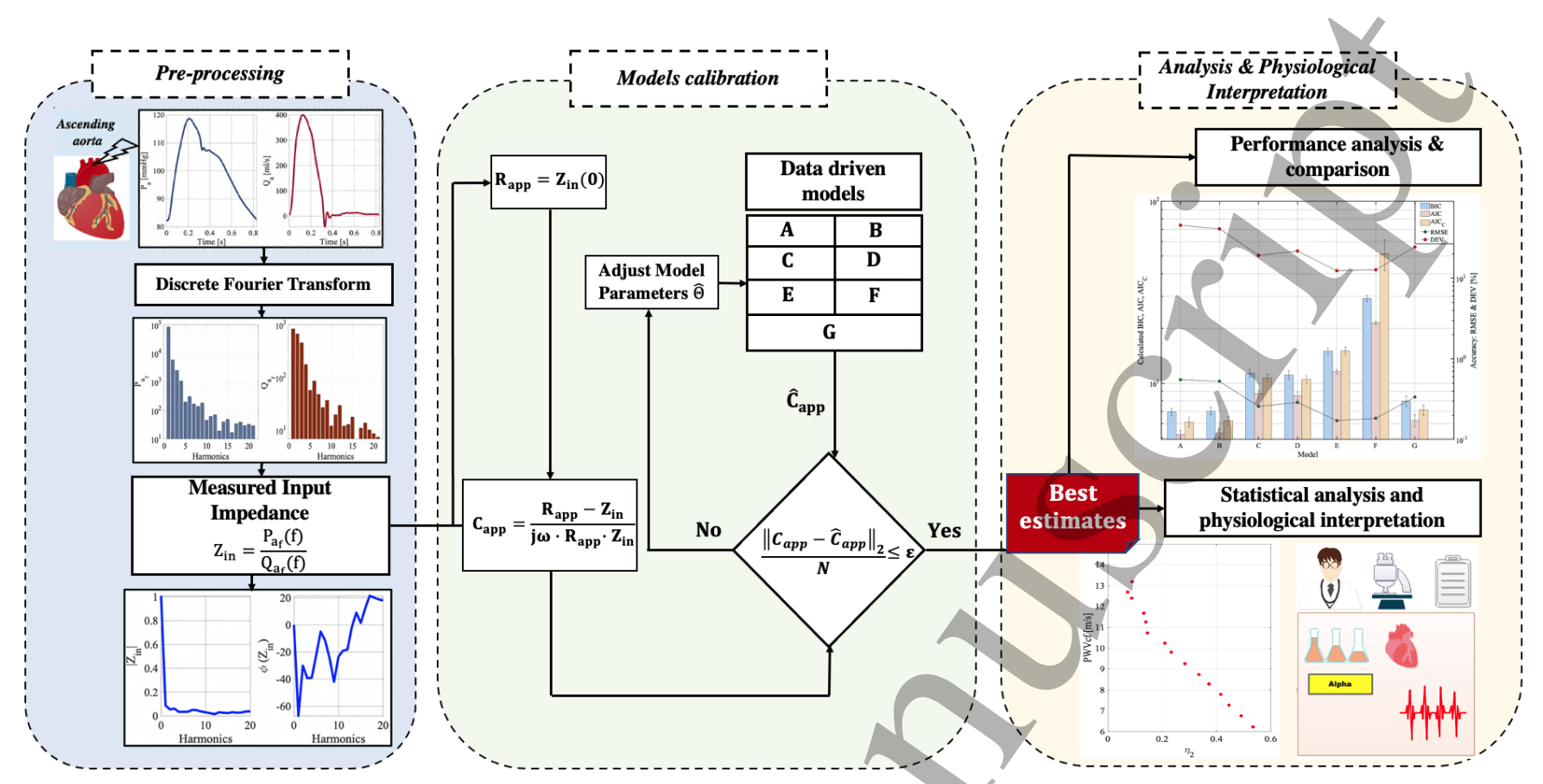

Fig. 5: A flowchart showing the three main steps of the calibration of the proposed fractional-order model representations of the apparent vascular compliance.

the simulated PWs and derived indices with corresponding in-vivo data. The generated PWs showed similar variations with age to those reported in in-vivo investigations, including changes in PW shape and in hemodynamic parameters derived from PWs.

3) Limitations of the investigated database: The main finding of the used in-silico data that the reproduced PWs exhibited similar changes to those observed in-vivo. Accordingly, it gives confidence that the approach generates realistic changes with age. Although these results showed a prominent potential in analyzing and the hemodynamic and characterizing vascular properties, it is worth noting that the used 1D model presents some limitations which should be taken into consideration when evaluating such in-silico investigation. Here, we list the main limitations as detailed in [42]:

- The input parameter explored in this approach are obtained from the previous study that included principally cross-sectional hemodynamic parameters rather than longitudinal. Accordingly, the differences in simulated PWs with respect to age can represent those that would be perceived between subjects of different ages, rather than those observed within an individual over time.

- Peter H.Charlton et al., [42], found insufficient evidence in the literature detailing how some cardiovascular properties vary with respect to age, namely, peripheral yascular compliance and the diameters of peripheral arteries. In addition, they found only minimal evidence to model the associations between certain parameters causing hemodynamic disorders.

- The develloped approach does not include a clear policy for adjusting the arterial network geometry with repect to the variation in height and body surface area. Also, the developed approach does not incorporate the sexassociated differences in hemodynamics which could yield to a relevant insight when analysing PWs features between females and males.

\section{B. Numerical implementation and Models calibration algo- rithm}

In this part, we present the general framework for identifying and calibrating the proposed model's parameters. The estimation approach was based on a non-linear least square minimization routine, using the well-known MATLAB - R2019b, function fmincon. This function is based on the trust-region reflective method. 5 illustrates the general framework used to validate the proposed fractionalorder model and compares its performance to standard models to estimate the apparent vascular compliance. Briefly, the algorithm can be separated into three main steps as follow:

1) Pre-processing step: In this step, the in-silico pressure, $P_{a}(t)$, and flow, $Q_{a}(t)$, at the level of the ascending aorta are collected. Then, these two hemodynamic signals are decomposed using Fast Fourier Transform (FFT), which translates them into a sum of their mean value and a series of sinusoidal components at a specific frequency, according 
to the following expressions:

$$
\begin{gathered}
P_{a}(t)=P_{a_{0}}+\sum_{n=1}^{N} P_{a_{n}} \cos \left(n \omega_{0} t+\beta_{n}\right), \\
Q_{a}(t)=Q_{a_{0}}+\sum_{n=1}^{N} Q_{a_{n}} \cos \left(m \omega_{0} t+\Theta_{n}\right) .
\end{gathered}
$$

In the above expressions, $P_{a_{0}}$ and $Q_{a_{0}}$ correspond to the mean value of the aortic pressure and flow respectively; $n$ indicates the $n^{\text {th }}$ harmonic of the flow and pressure in the Fourier series; $N$ is the total number of harmonics; $P_{a_{n}}$ and $Q_{a_{n}}$ are the amplitudes of the $n^{\text {th }}$ sinusoidal component of the aortic pressure and flow, respectively, and $\beta_{n}$ and $\Theta_{n}$ are the related phase angles; $\omega_{0}$ is the fundamental frequency of the cardiac pulsation in the unit of $[\mathrm{rad} / \mathrm{sec}]\left(\omega_{0}=2 \pi / T\right.$ where $T$ is the heart period). Each term $P_{a}(j n w)$ and $Q_{a}(j n w)$ obtained by FFT can be expressed as function of its modulus, $P_{a_{n}}$ and $Q_{a_{n}}$ and its phase angle, $\beta_{n}$ and $\Theta_{n}$ as follows:

$$
\begin{aligned}
P_{a}\left(j n w_{0}\right) & =P_{a n}\left[e^{j\left(n \omega_{0} t+\beta_{n}\right)}\right], \\
Q_{a}\left(j n w_{0}\right) & =Q_{a n}\left[e^{j\left(n \omega_{0} t+\Theta_{n}\right)}\right] .
\end{aligned}
$$

Subsequently, harmonic by harmonic, the in-silico aortic input impedance is evaluated as the ratio of $P_{a}\left(j n w_{0}\right)$ to $Q_{a}\left(j n w_{0}\right)$ :

$$
Z_{\text {in }}(j n w)=\frac{P_{a n}}{Q_{a n}} \frac{\left[e^{j\left(n \omega_{0} t+\beta_{n}\right)}\right]}{\left[e^{j\left(n \omega_{0} t+\Theta_{n}\right)}\right]}=Z_{n} e^{j \Phi_{n}}
$$

where $\Phi_{n}$ is the phase angle of $Z_{\text {in }}$ as the difference between $\beta_{n}$ and $\Theta_{n}$, and $Z_{n}$ is its modulus as the ratio between $P_{a n}$ and $Q_{a n}$.

2) Model-calibration step: After preparing the in-silico input impedance, the subsequent step is to calculate the in-silico apparent compliance $C_{a p p}$. Accordingly, we first calculate the apparent resistance $R_{a p p}$, which is supposed to be a constant, corresponding to the $D C$ component of the aortic input impedance as $R_{a p p}=P_{a_{0}} / Q_{a_{0}}=Z_{i n}(0)$. Then, we evaluate the in-silico apparent compliance using equation (4). Subsequently, we fit the prepared apparent compliance data to one equation of the proposed model's representation as described in the Model's section. The parameters to estimate for each model's representation $C_{c}^{\text {Model }}$ are refereed as $\Theta^{\text {Model }}$ where Model $=\{(A),(B),(C),(D),(E)\}$ corresponds to the index of the model's representation:

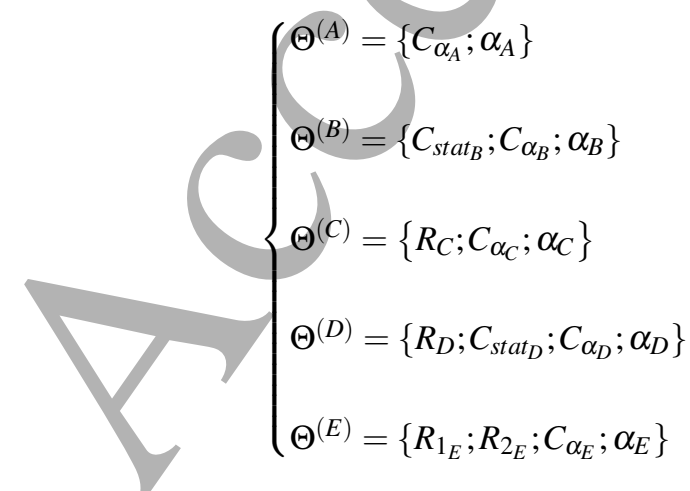

The estimate of $\Theta^{\text {Model }}$ is $\hat{\Theta}^{\text {Model }}$ were found via the solution of the inverse problem of the estimated apparent compliance $\left(\hat{C}_{a p p}=C_{c}^{\text {Model }}(\Theta)\right)$ and the in-silico one $\left(C_{a p p}\right)$. Initialized by $\theta_{0}$, the inverse algorithm iteratively predicts the set of parameters $\hat{\theta}$ which minimizes the root mean square error (RMSE) between the complex $C_{a p p_{[i]}}$ and the model predicted $\hat{C}_{a p p_{[i}}(\theta)$ evaluated at the $i^{\text {th }}$ harmonic. The objective function RMSE is defined as:

$$
R M S E=\sqrt{\frac{\sum_{i=1}^{N_{s}}\left(\frac{\operatorname{Re} e_{i}-\operatorname{Re} \hat{(\Theta)}}{\max \left(\operatorname{Re}_{i}\right)}\right)^{2}+\left(\frac{\operatorname{Im}_{i}-\operatorname{Im}_{i}(\Theta)}{\operatorname{Im}_{i}}\right)^{2}}{N_{s}}}
$$

Where $N_{s}$ denoting the number of excited frequency points, $R e_{i}$ and $I m_{i}$ denoting the real and imaginary parts of the insilico $C_{a p p}$, and $\mathrm{Im}$, and $\hat{\hat{e}_{i}}$ and $I \hat{m}_{i}$ designate the real and imaginary parts of the model of $C_{a p p}$, respectively. $\hat{\theta}$ are the estimates that minimize RMSE.

$$
\hat{\Theta}=\arg \min _{\Theta} R M S E
$$

In the above function, we constrained all parameters to be positive to guarantee physical properties. Once a tolerance of error was reached, the convergence of the method is confirmed, the fmincon function exits and yields an output of the optimal set of model parameters estimates $\hat{\theta}^{*}$.

3) Models comparison and statistic: In this study, we compare the performance of the proposed model with their corresponding integer-order version, where the fractional differentiation order $\alpha$ is equal to 1 . Accordingly, the integer order version of model (A) will be equivalent to an ideal capacitor whose capacitance is a constant, which is not frequency-dependent, hence in our comparison, we exclude this case. Similarly, the integer-order version of model (B) leads to a series of two ideal capacitors, which is as well, equivalent to an ideal capacitor whose capacitance is constant. For the models, (C) and (D), the integer-order version is equivalent to the analog Voigt cell model (an ideal capacitor connected in series with a resistor). Conclusively, in this work, we compare our proposed methods to the Voigt cell model. On top of that, in order to show the role of fractionalorder concept in reducing the complexity of such approach, we conduct an extra comparison with the well-known general apparent compliance-based model for viscoelastic material [64] which is expressed as:

$$
C_{c}^{F}=C_{s t a t} \frac{\prod_{n=1}^{N} a_{n}\left(j \omega+b_{n}\right)}{\prod_{n=1}^{N} b_{n}\left(j \omega+a_{n}\right)},
$$

where $a_{n}$ and $b_{n}$ are imperial constants that can be convenient to fit any particular case. $C_{\text {stat }}$ denoting the static compliance for the vessel. Goedhard et. all showed that this model could fit an experimental data with $N=4$. Hence in our comparison we choose $N=4$. We refer to the viscoelastic model and Voigt model as models (F) and (G), respectively.

Because the proposed models have a different number of parameter, to perform a fair comparison, along with the 


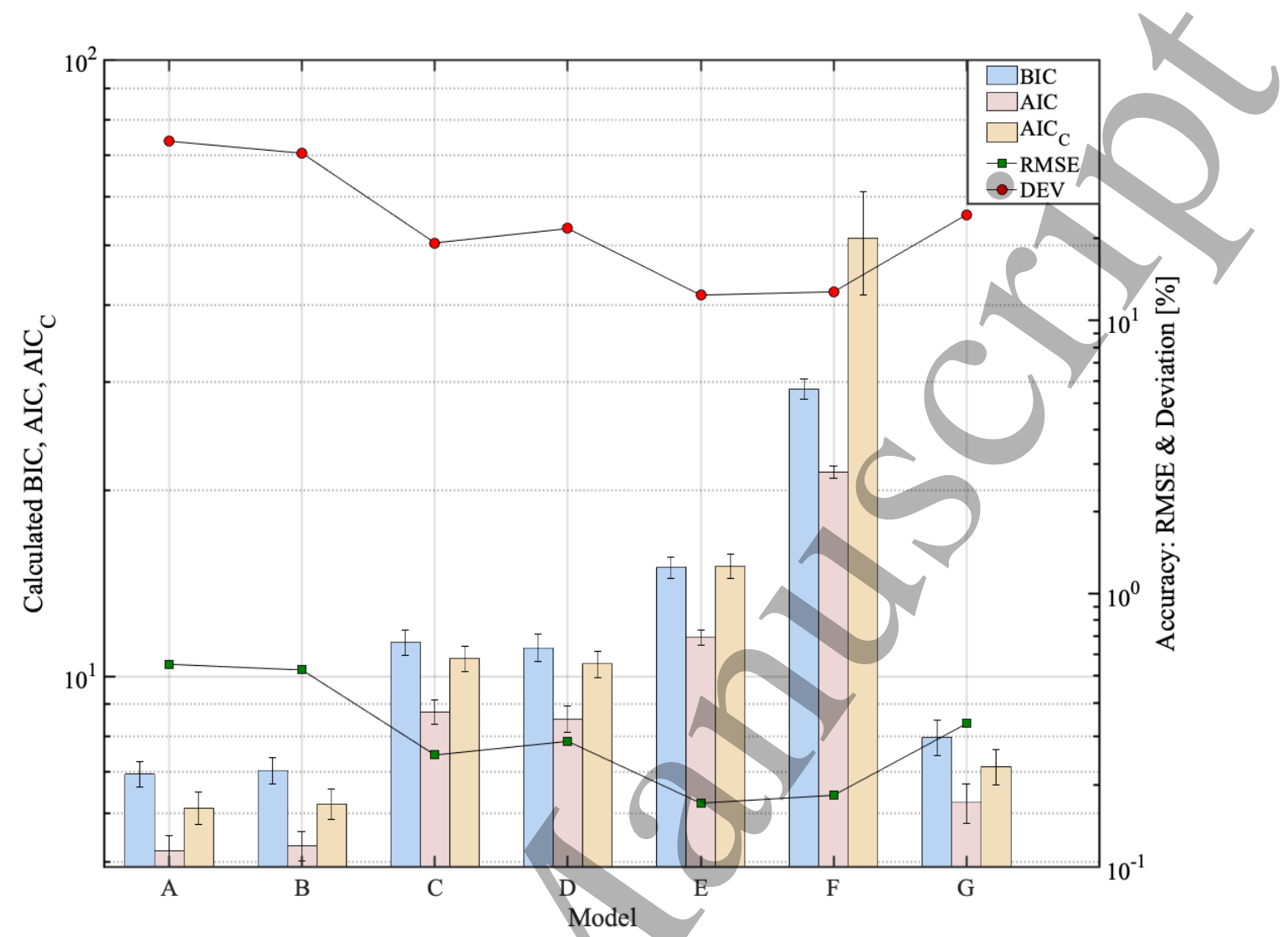

Fig. 6: A plot of the mean values of the goodness of fit criterion RMSE and Deviation[\%] represented in the right y-axis along with a bar chart of the calculated $B I C, A I C$, and $A I C_{C}$, represented in the left y-axis, after fitting all the in-silico data to the proposed fractional-order models and the standard ones. The error bars based on standard deviation values of the calculated $B I C, A I C$, and $A I C_{C}$ are also shown.

RMSE, we evaluate the Bayesian Information Criterion/following equation: $(B I C)$, Akaike Information Criterion, $\left(I C_{C}\right)$, and the corrected Akaike Information Criterion $\left(A I C_{C}\right)$ that are defined as follows:

$$
\left\{\begin{array}{l}
B I C=-2 \cdot \ln (R M S E)+P \cdot \ln \left(N_{S}\right) \\
A I C=-2 \cdot \ln (R M S E)+2 \cdot P \\
A I C_{C}=-2 \cdot \ln (R M S E)+\frac{2 \cdot P \cdot N_{S}}{N_{S}-P-1}
\end{array}\right.
$$

where $\mathrm{P}$ is the number of parameters. Furthermore, the deviation of the model modulus from the in-silico apparent compliance modulus was calculated, using the following expression:

$$
D_{i}[\%]=\left[\frac{\left|C_{c_{[i]}^{\text {model }} \mid}\right|-\left|C_{a p p_{[i]}}\right|}{\left|C_{a p p_{[i]}}\right|}\right]_{i=1 . . N_{s}} \times 100 \% .
$$

For ease of visualization of the various comparisons between the different models, for each virtual subject, we evaluated the mean of $\mathrm{D}[\%]$ over the $N_{s}$ harmonics, based on the

$$
\text { Deviation }[\%]=\frac{\sum_{i=1}^{N_{s}} D_{i}[\%]}{N_{S}} .
$$

\section{RESULTS}

\section{A. Quantifying the models performances}

The mean values of the goodness of fit criterion $R M S E$ and Deviation $(\%)$ as well as the calculated $\{B I C, A I C$, and $\left.A I C_{C}\right\}$, after fitting the whole in-silico data to the proposed fractional-order models and the standard ones, are presented in TABLE II Fig. 6, shows the bar chart of these parameters along with their error bars that correspond to their standard deviation. For the ease of visualization, the RMSE and Deviation (\%) mean values versus models are added to this plot. Additionally, we reported in TABLE IV and TABLE $\mathrm{V}$, in Appendix C, all these criterion's mean values for each group of age as well as heart rate subgroup of the in-silico data.

As the proposed representations have different number of parameters, in order to perceive the compromise between complexity and accuracy and compare between the proposed paradigms, we present in Fig. 7, a radar chart that combines all the performance variables of all the methods in one graph. The axis of this chart correspond to the evaluated $\{B I C$; $A I C$; and $A I C_{c} ; R M S E ;$ Deviation[\%]\}. As for any modeling scheme, based on the these results, it is clear that there is a 
TABLE II: Mean values of the calculated $B I C, A I C$, and $A I C_{c}$ and the goodness of fit criterion $R M S E$ and Deviation[\%]

\begin{tabular}{|c|c|c|c|c|c|c|c|}
\cline { 2 - 7 } \multicolumn{1}{c|}{} & Model (A) & Model (B) & Model (C) & Model (D) & Model (E) & Model Model (F) & Model (G) \\
\hline BIC & $6.94 \pm 0.34$ & $7.04 \pm 0.34$ & $11.35 \pm 0.52$ & $11.12 \pm 0.57$ & $15.01 \pm 0.6$ & $29.23 \pm 1.12$ & $7.96 \pm 0.52$ \\
\hline AIC & $5.21 \pm 0.31$ & $5.31 \pm 0.3$ & $8.75 \pm 0.39$ & $8.53 \pm 0.42$ & $11.55 \pm 0.33$ & $21.45 \pm 0.45$ & $6.24 \pm 0.46$ \\
\hline AIC $C$ & $6.11 \pm 0.37$ & $6.2 \pm 0.36$ & $10.7 \pm 0.51$ & $10.47 \pm 0.51$ & $15.1 \pm 0.7$ & $51.4 \pm 9.76$ & $7.13 \pm 0.47$ \\
\hline RMSE & $0.55 \pm 0.08$ & $0.53 \pm 0.08$ & $0.26 \pm 0.05$ & $0.29 \pm 0.05$ & $0.17 \pm 0.03$ & $0.18 \pm 0.04$ & $0.33 \pm 0.07$ \\
\hline Deviation [\%] & $45.41 \pm 11.74$ & $41.03 \pm 9.85$ & $19.27 \pm 5.12$ & $21.74 \pm 5.9$ & $12.41 \pm 2.78$ & $12.76 \pm 2.94$ & $24.45 \pm 6.72$ \\
\hline
\end{tabular}

good compromise between the accuracy and complexity of the model. Among the five proposed fractional-order model, the single fractional-order capacitor-based representation of the apparent vascular compliance, $\operatorname{Model}(\mathrm{A})$, fails to produce the lowest RMSE and Deviation for any of the data sets; however, it represents the smallest $\left\{B I C, A I C\right.$, and $\left.A I C_{c}\right\}$. On the contrary, the fractional-order representation, Model (E) and the integer-order representation Model (F) exhibit the lowest Deviation and RMSE at the expense of the complexity that has been reflected in the high values of $\{B I C, A I C$, and $\left.A I C_{c}\right\}$. It is worth to mention that the fractional-order representation, Model $(\mathrm{E})$, comprises only four parameters and performed better than Model (F), which posses nine parameters. By checking the remaining models' performances, it is subgroup. clear that the representations, Model (C) and Model (D) and Model (G), well compromise between the complexity and the accuracy. However, in terms of accuracy performance, among the latest models, the fractional-order representation,
Model (C), can be considered as the optimal representation. Its $R M S E$ is around $0.26 \pm 0.05$. Besides, it presents an $A I C_{c}$ equals $10.7 \pm 0.51$, which is approximately five times lower than the representation of Model (F). Conclusively, from this analysis, it is apparent that model system fractionalizing is enhancing the accuracy of the arterial compliance as well as reducing the complexity.

\section{B. Statistical Analysis of the estimated parameters}

In this section we present the statistical analysis of the estimated parameters of the proposed fractional-order representations $\{$ Model (A); Model (B); Model (C); Model (D); Model (E); \}. The mean value of the parameter estimates of these model representations are reported in TABLE VI, in the appendix $\mathrm{C}$, for each group of age and heart rate

1) Model (A): The sub-figure 8(A) shows the distribution of the parameter estimates of Model (A), after fitting the in-silico data of the arterial compliance. By observing

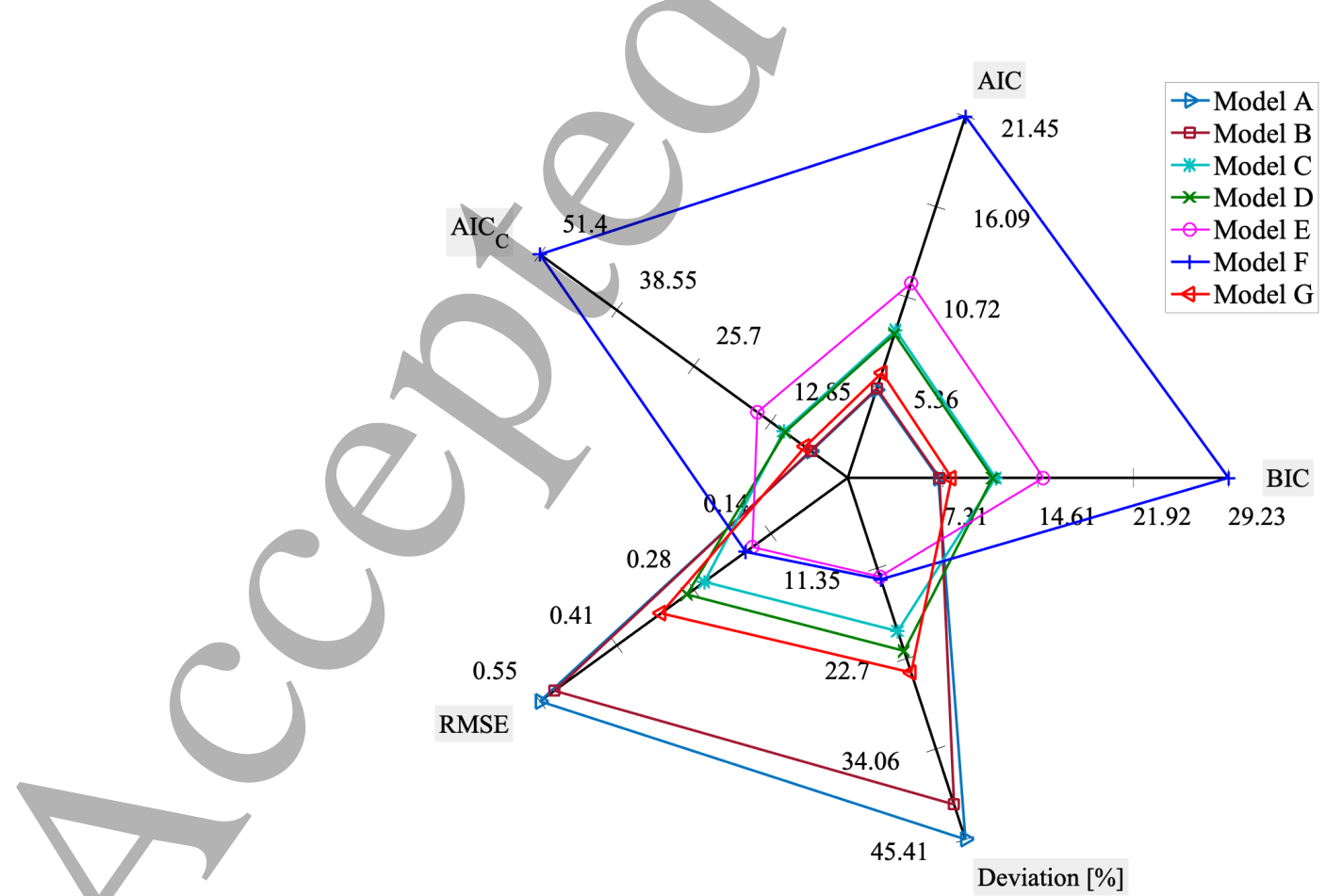

Fig. 7: Radar chart summarizes all the models goodness of fit performances along with the the calculated $B I C, A I C$, and $A I C_{c}$ criterion, in one graph. 

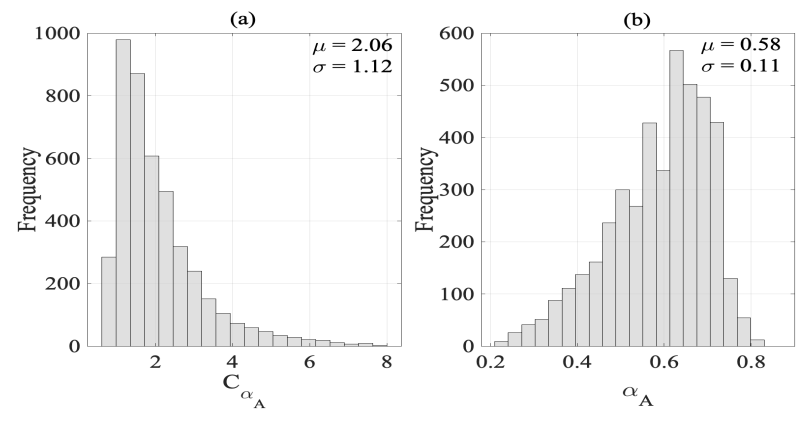

(A) Distribution, mean value and standard deviation of Model A parameter estimates: (a) the pseudo-capacitance $C_{\alpha_{A}}$, and (b) the fractional differentiation order parameter $\alpha_{A}$.

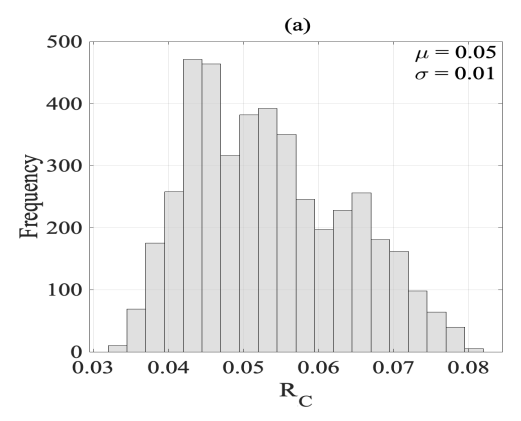

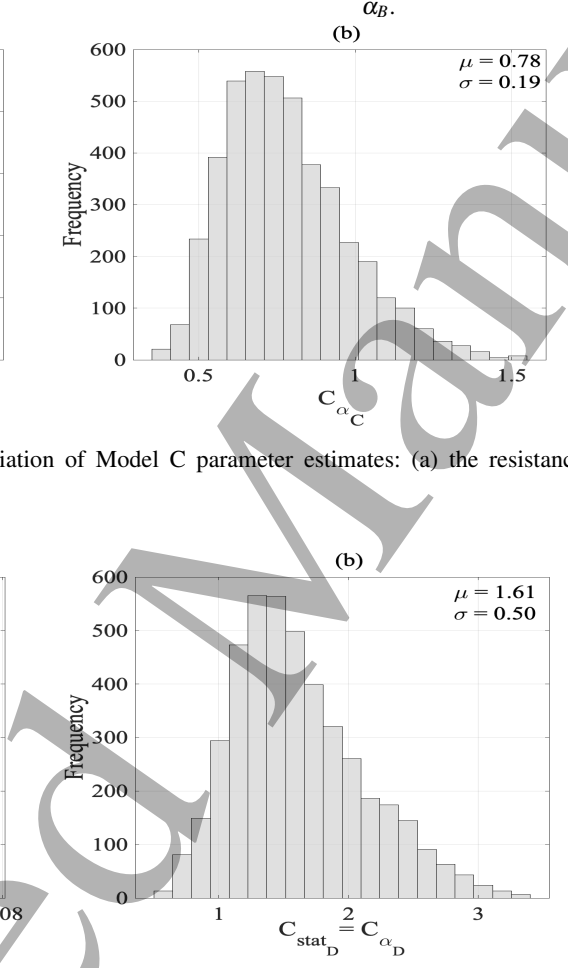
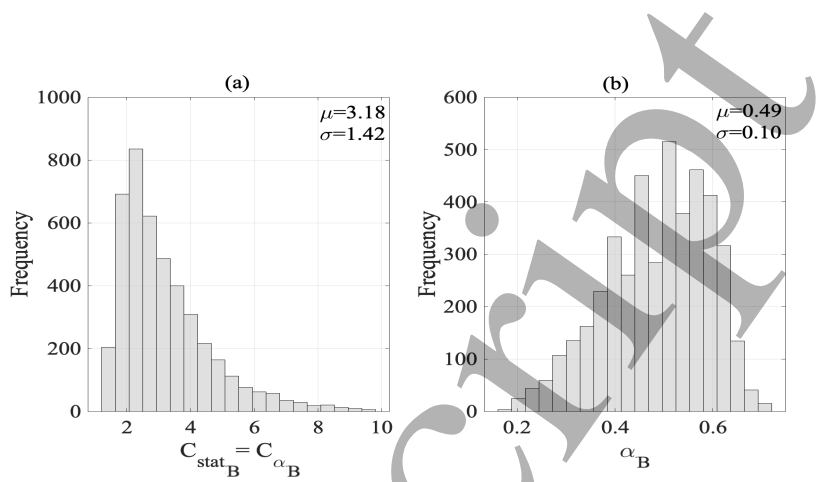

(B) Distribution, mean value and standard deviation of Model B parameter estimates: (a) the static capacitance $C_{\text {stat }}$ and the pseudo capacitance $C_{\alpha_{B}}$ which are equals, and (b) the fractional differentiation order parameter $\alpha_{B}$.

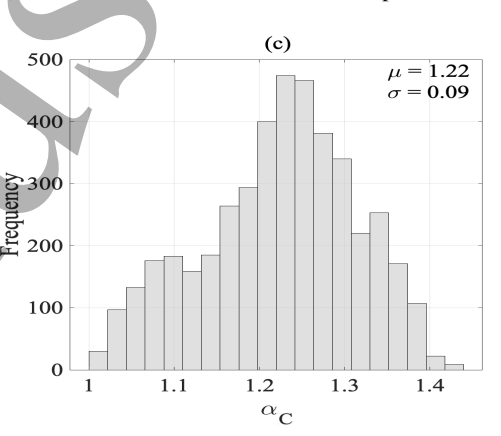

(C) Distribution, mean value and standard deviation of Model C parameter estimates: (a) the resistance $R_{C}$, (b) the pseudo-capacitance $C_{\alpha_{C}}$, and (c) the fractional differentiation order parameter $\alpha_{C}$.
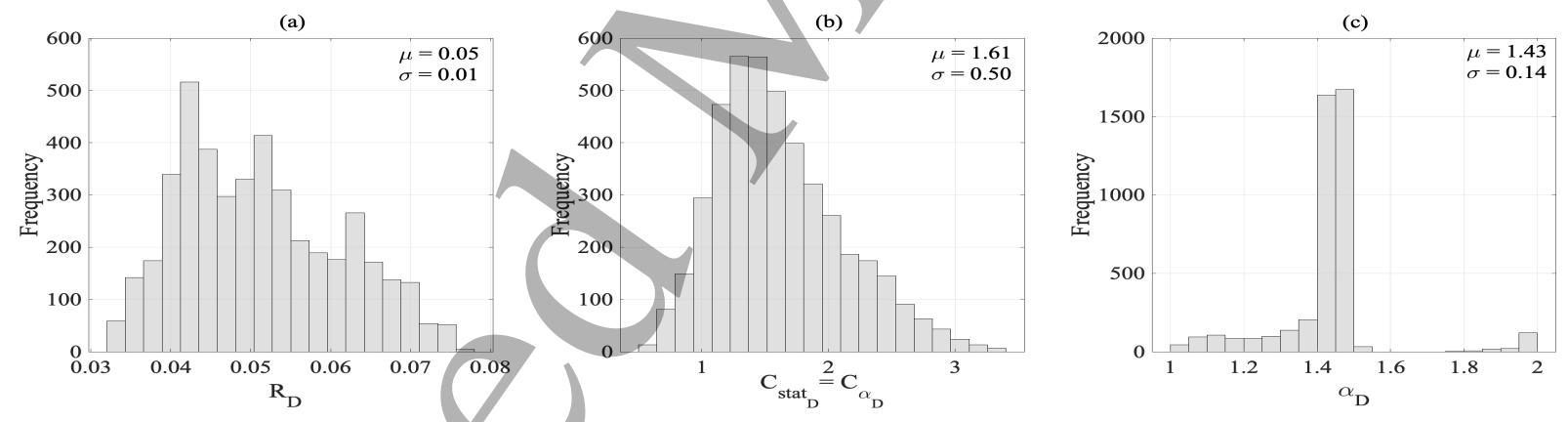

(D) Distribution, mean value and Standard deviation of Model D parameter estimates: (a) the resistance $R_{D}$, (b) the static capacitance $C_{\text {stat }}$ and the pseudo capacitance $C_{\alpha_{D}}$ which are equals, and (c) the fractional differentiation order parameter $\alpha_{D}$.
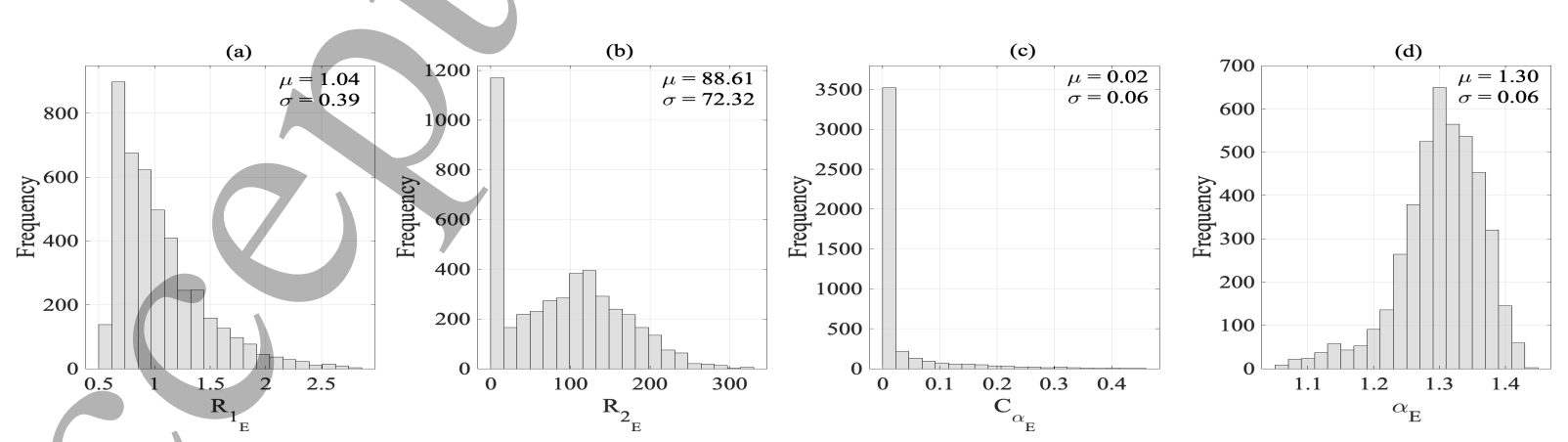

(E) Distribution, mean value and standard deviation of Model E parameter estimates: (a) the resistance $R_{1_{E}}$, (b) the resistance $R_{2}$, (c) the pseudo capacitance $C_{\alpha_{E}}$, and (d) the fractional differentiation order parameter $\alpha_{E}$.

Fig. 8: presents the distribution, mean value and standard deviation of the parameters estimates of the proposed fractional-order model representations of apparent vascular compliance 
the distribution of the fractional differentiation order, $\alpha$, estimates, it is clear that this parameter is less than 1 for all the subjects. Its mean value is approximately $0.58 \pm 0.008$. It is worth noting that in the estimation phase, for the parameter $\alpha$, we have only constrained the lower bound to be zero; however, for the upper bound, it was unconstrained. Accordingly, this result indicates that the arterial system exhibits a viscoelastic behavior, not a purely elastic one. Indeed, the fact that $\alpha \neq 1$ implies that the FOC element incorporates both resistance and capacitance behaviors, as demonstrated mathematically in (10). This result further supports the concept of fractional-order behavior by the arterial system. In the proposed model, the fractional-order element combines both the resistance and the capacitance properties, which display the viscoelastic behavior of the arterial vessel. The contributions from both properties are controlled by the fractional differentiation order $\alpha$, enabling a more flexible physiological description. As the fractional power approaches to 1 , the capacitance part dominates and, hence the arterial system behaves like a pure elastic system.

2) Model (B): The sub-figure 8(B) shows the distribution of the parameter estimates of the Model (B) after fitting the in-silico data of the arterial compliance. In this model, the static capacitance has been chosen to be equal to the pseudo-capacitance of FOC, $\left(C_{\alpha_{B}}=C_{s t a t_{B}}\right)$. By observing the distribution of $\alpha_{B}$, we can notice that this parameter is less than 1 for all the population with a mean value equal to $0.49 \pm 0.10$. Comparing to Model $(\mathrm{A})$, the fractional factor has been decreased by approximately 0.1 ; however, the pseudo-capacitance was increased by 1 . The decrease of $\alpha$ implies an increase of the effective resistive part of the FOC, as explained in the previous parts, which comes as to compensate for the increase in the overall capacitive part of the whole system model. In this model, the ideal capacitor is counting for static compliance, whereas the fractional-order one controls the arterial stiffness levels. In other words, $\alpha$ might give a piece of information about the variation of the viscoelasticity of the arteries.

3) Model $(C)$ : The sub-figure 8(C) shows the distribution of the parameter estimates of the Model B after fitting the in-silico data of the arterial compliance. By observing the distribution of $\alpha_{C}$, it is clear that for all the subjects, this parameter is higher than 1 with a mean value of approximately equals to $1.22 \pm 0.09$. Mathematically, as $\alpha$ exceeds 1 , the real part of the fractional-order element impedance, $\mathrm{G}_{\mathrm{r}}$, becomes negative, and hence it has the characteristic of a negative resistor that supplies power. Having a negative resistance, in this case, comes as compensation for the added series resistance $R_{C}$. Besides, comparing to Model (A), it is worthy to notice that the mean value of the pseudo-capacitance $C_{\alpha_{C}}$ was decreased to $0.78 \pm 0.19$.
4) Model (D): The sub-figure 8 (D) shows the distribution of the parameter estimates of the Model $D$ after fitting the in-silico data of the arterial compliance. This model incorporates a static capacitor in series to FOC, along with a small resistor. Comparing to $\operatorname{Model}(\mathrm{B})$, the addition of the small resistance causes $\alpha$ to go beyond 1 with a mean value approximately equal to $1.43 \pm 0.14$. In fact as introduced previously, $(\alpha>1)$ means that the real part of the FOC's impedance, $\mathrm{G}_{\mathrm{r}}$, becomes negative. Besides, the mean value of static compliance and pseudo-capacitance decreases to $1.61 \pm 0.50$. In this configuration, the addition of serial constant resistor and capacitor in this model is for the sake of accounting for the static viscosity and elasticity, respectively, while FOC depicts the ability of the arterial vessel to store blood dynamically.

5) Model (E): The sub-figure $8(\mathrm{E})$ shows the distribution of the parameter estimates of the Model (E) after fitting the in-silico data of the arterial compliance. This model is similar to the equivalent analog circuit of Maxwell's mechanical element ( series spring and dashpot in parallel with a dashpot), whereas instead of using an ideal capacitor to represent the spring, FOC has been employed. In terms of performance, Model (E) is the best. Similar to all the proposed model, $\alpha_{D} \in \mathbb{R}$ which demonstrate the fractionalorder behavior of the apparent arterial compliance. By observing the distribution of $R_{1_{E}} R_{2_{E}}$, it is noticeable that these parameters are larger than $R_{B}$ and $R_{D}$.

\section{Discussion}

The accuracy and simplicity criteria in developing model representations for the estimation of the apparent vascular compliance are necessary; however, they are not sufficient. Fundamentally an ideal estimation approach should also meet the condition of interpretability and explain-ability that can be satisfied as follow:

- The approach should have a foundation in the basics physics

- The approach does not assume critical unknown information. It should only be limited by the nature and quality of the data, not the model's assumptions.

- The model extracts information from the most relevant portion of the data. Accordingly, it consists of parameters representing physiological attributes, serving in interpreting the physical processes, and matching with the experimental observations.

As we introduced in the previous section, the use of fractional-order tools, such as FOC, for the vascular system characterization has been justified in many experimental studies. Besides, it has been demonstrated that that fractional-order framework is more suitable for probing the viscoelasticity of collagenous tissues in the arterial bed [28]. In fact, fractional-order framework allows the reduction of the complexity order and further improve the modeling accuracy. In addition, In-vivo investigations have shown 
that the fractional-order differentiation order, $\alpha$, is a key parameter as it is associated with smooth muscle (SM) activity that modulates the viscoelasticity in arteries. In fact, SM cells stretch collagenous fibres and hence, vascular activation modifies the local viscoelastic response of the arterial wall [28]. Besides, a clear power-law response was shown in the frequency response to estimate Young's modulus in the range $0-100 \mathrm{~Hz}$ [65], [29].

Bearing these findings in mind, the physical basis of the proposed approaches that incorporate FOC is warranted. In the following, we check the relationship and correlations between the additional novel fractional-order parameter $(\alpha)$ and the associated hysteresivity coefficient $\left(\eta_{r}\right)$ and the main determinant of the central hemodynamic as well as the central and carotid-femoral pulse-wave velocity indices. The ultimate goal is to associate to these novel parameters a clear physiological attribution so they can be invested as surrogates or mathematical biomarkers of certain physiological indices usually difficult to extract and interpret they develop.

\section{A. Relations between fractional-order parameters and cen- tral hemodynamic characteristics}

Several research studies have observed that the changes in the determinants of the central blood pressure waveform, such as systolic blood pressure SBP, diastolic blood pressure DBP, and pulse pressure (APP), are strongly associated with cardiovascular diseases incidents. For instance, the augmentation of the SBP or APP is considered as a reflection marking the improper functioning of the cardiovascular system. In fact, stiffer arteries resulting from the arteriosclerosis disease causes increases in the SBP as well as arterial pulse wave velocity $(P W V)$. PWV, such as carotid-to-femoral one $\left(P W V_{c f}\right)$, are recognized as valuable surrogates of the arterial stiffness. In this part, we investigate whether the fractional differentiation order, $\alpha$, and the hysteresivity coefficient, $\eta_{r}$, (defined by (11)) correlate with the central blood pressure determinants and the arterial pulse wave velocity $\left(P W V_{a}\right)$ and $\left(P W V_{c f}\right)$. In addition to the arterial pulse waves, the used database provides both $\left(P W V_{a}\right)$ and $\left(P W V_{c f}\right)$ for each subject.

Accordingly, to/evaluate the associations between the fractional-order parameters and the central hemodynamic determinants, for each model, we calculated the average value of $\alpha$ and $\eta_{r}$ estimates over a fixed interval of the blood pressure determinant (SBP, DBP, and APP) that is equal to $5[\mathrm{mmHg}]$ and PWV that is equal to $0.5[\mathrm{~m} / \mathrm{s}]$. TABLE IIII shows the correlation coefficients between $\alpha$, and $\eta_{r}$ and (SBP, DBP, APP, $\mathrm{PWV}_{\mathrm{a}}$ and $\left.\mathrm{PWV}_{\mathrm{cf}}\right)(95 \%$ confidence interval). In addition, we present, in Fig. 9 the scattergrams of $\alpha$ of each model versus aortic systolic blood pressure (SBP), aortic diastolic blood pressure (DBP), aortic pulse pressure (APP), aortic pulse wave velocity $\left(P W V_{a}\right)$ and carotid-femoral pulse wave velocity $\left(P W V_{c f}\right)$. Each row corresponds to a fractional-order model representation. Similarly, Fig. 10 illustrates the scattergrams of $\eta_{r}$ with respect to the hemodynamic determinants for each model representation.

It is clear from these results that, for the majority of the models, the fractional-order parameters are strongly associated with the hemodynamic determinants. For instance, with regards to SBP, DBP, and APP, we notice that for all the proposed model, excepting for Model (D) the correlation coefficients with respect to $\eta_{r}$ and $\alpha$ are larger or equal to 0.90. Regarding to the stiffness indexes, $\left(P W V_{a}\right)$ and $\left(P W V_{c f}\right)$, the coefficients correlation are approximately equal or larger than 0.85 for all the proposed model apart from Model (D). Overall, the fractional-order parameter estimates of Model (C) present the best correlation coefficients. This result is in agreement with the goodness of the fit performance of this model. In fact, as analyzed in the previous parts, Model (C) provides a compromise between the accuracy and complexity of other proposed fractional-order models. In addition, although Model (A) is not very accurate in estimating the apparent compliance and not complicated, the correlation coefficients between its parameter estimates and the hemodynamic determinant as well as the central PWVs 

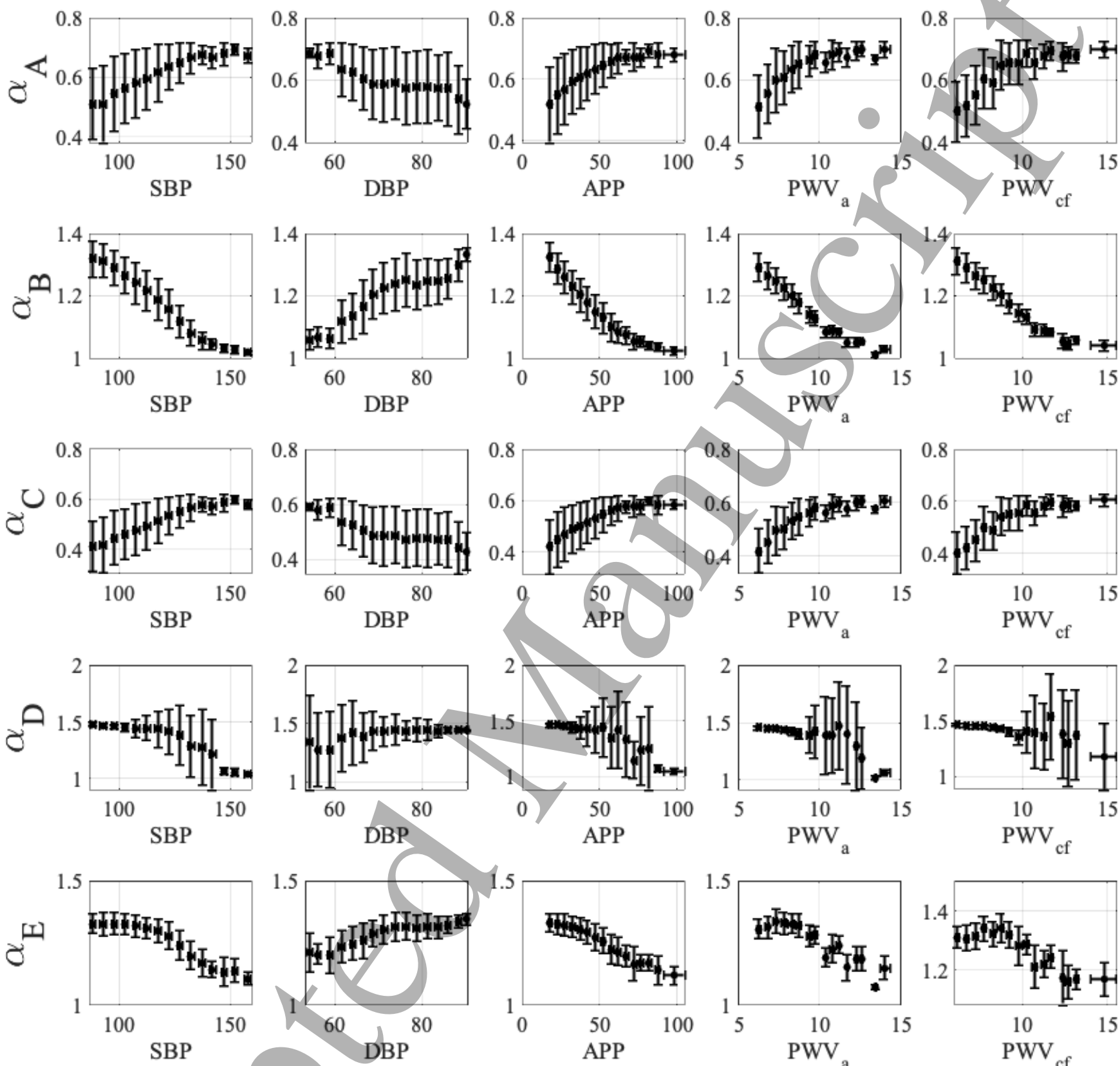
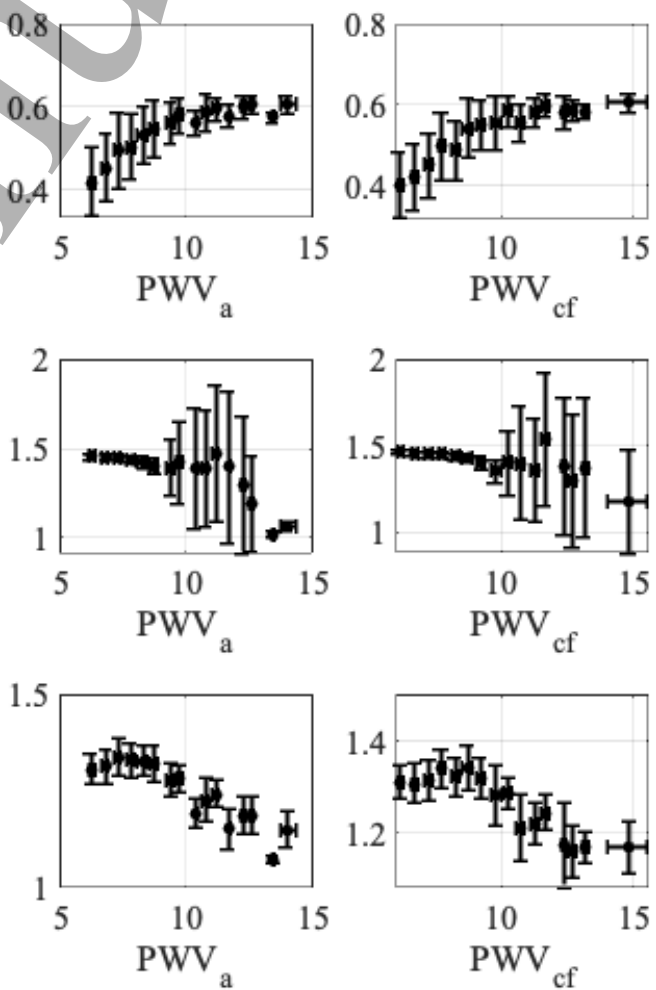

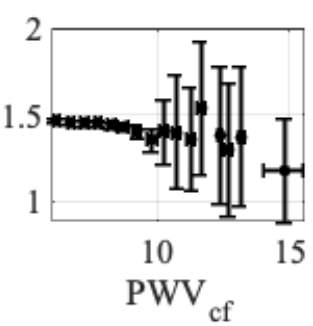

Fig. 9: illustrates the scattergrams of of the fractional-order parameter $\alpha$ of each model versus aortic systolic blood pressure (SBP), aortic diastolic blood pressure (DBP), aortic pulse pressure (APP), aortic pulse wave velocity $\left(P W V_{a}\right)$ and carotid-femoral pulse wave velocity $\left(P W V_{c f}\right)$. Each row corresponds to a fractional-order model representation.

are acceptable and reasonable.

Conclusively, our findings point out the potential interests of using $\mathrm{FOC}$ in the characterizing of arterial compliance. In addition, it demonstrates the viability of the fractional-order differentiation order to serve as a surrogate measure of the arterial stiffness or marker of cardiovascular diseases. Indeed, by assessing the fractional factor, $\alpha$, it is easy to evaluate the hysteresivity coefficient $\eta_{r}$ reflecting the ratio between two physiologically insightful parts: the tissue resistance and elastance.

\section{B. Limitations \& next steps for the fractional modeling}

The fractional-order paradigm proposed in this work should be developed a little further before its generalization in the hemodynamic modeling context. In fact, It is worthy to note the limitations of this study.

- In this work, due to the non-availability of real data, we used in-silico data. Although this database mimics the real physiological human states, and it is based on a validated one-dimensional numerical model of 

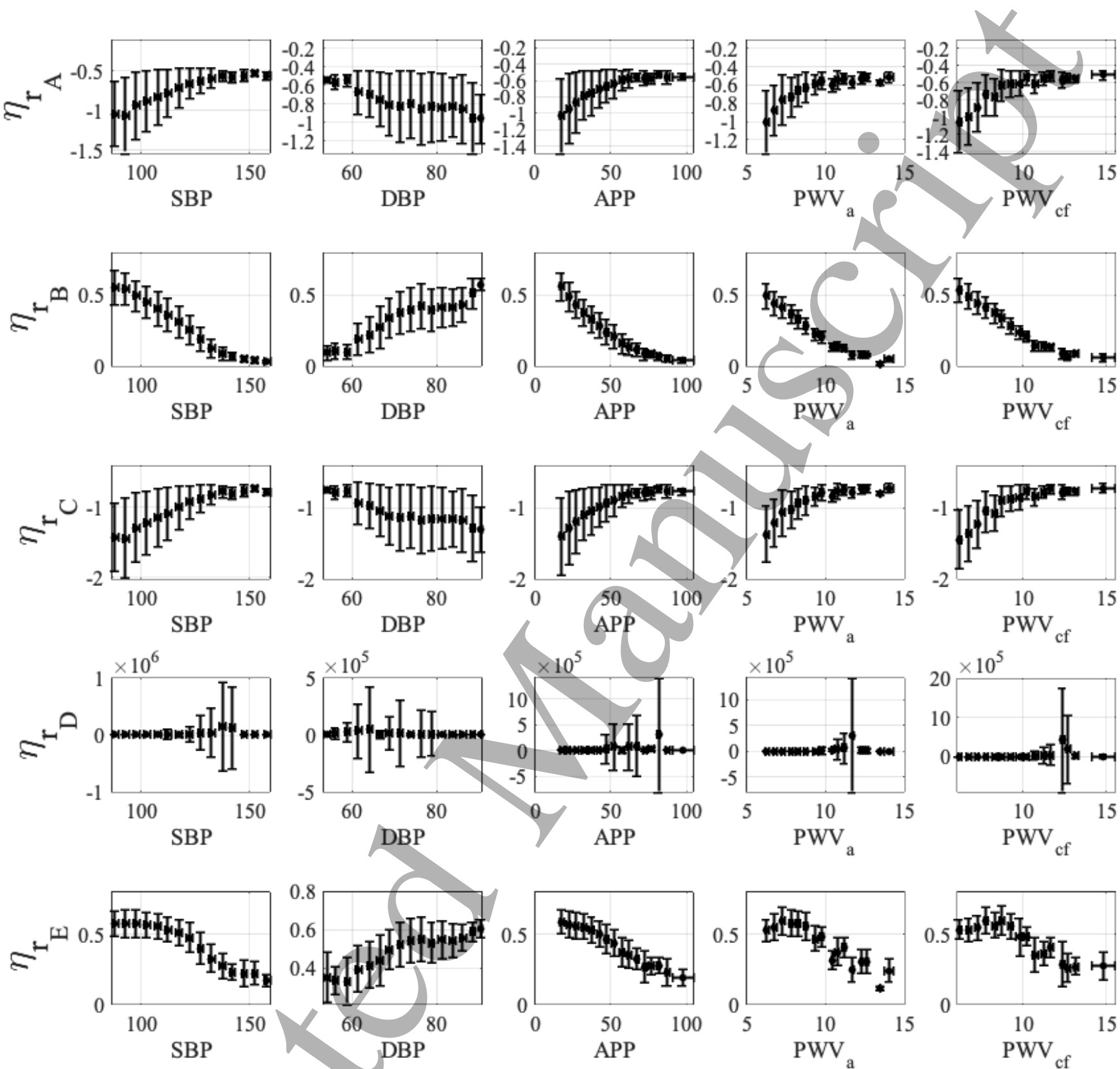

Fig. 10: illustrates the scattergrams of the hysteresivity coefficient $\eta_{r}$ of each model versus aortic systolic blood pressure (SBP), aortic diastolic blood pressure (DBP), aortic pulse pressure (APP), aortic pulse wave velocity $\left(P W V_{a}\right)$ and carotid-femoral pulse wave velocity $\left(P W V_{c f}\right)$. Each row corresponds to a fractional-order model representation.

the arterial network, in-vivo investigations are required to validate and verify the reliability of the proposed models. The use of real data would considerably give more credibility to the new paradigm.

- The estimation was based on only one cardiac cycle. Future work should derive metrics from multiple cycles of real data. This will help to assess and take into account the inter-beat interval variability. In addition, any experimental conditions and protocols should be adapted to generate the low-frequency component of the data. This will allow the testing and validation of the whole physiological spectrum range.

- The presented approaches should be conducted in a range of different real physiological situations and show a good fitting for all the cases. It is straightforward to use FOC in the simple model representation proposed here, but there is no explicit agreement on the exact physiological relevance of the new parameter, the fractional differentiation order $\alpha$ or $\eta_{r}$. Although it is evident from mathematical equations that $\alpha$ value controls the viscosity as well as the elasticity levels, it would be of great potential for clinical application, to define ranges 
of the $\alpha$ value for normal and pathological physiological conditions.

- It is well documented that arteries have viscoelastic properties. In the present work, this phenomenon is reflected in the complex frequency response, which has been captured mainly by the fractional-order element whose equivalent capacitance is frequency-dependent. However, arteries also exhibit nonlinear elastic response, which is very important [11]. Accordingly, it would be of great potential to incorporate the nonlinear elasticity vascular property within the fractional-order model representation, in the future investigations.

- This study does not consider the noise effect on the pulse wave signals. In fact, several sources of noise can be allocated with the blood pressure signal, such as the movement artifacts, poor sensor contact, and optical interference, etc. Accordingly, considering the noise can impact the utility of the estimation of the fractionalorder parameters. In the future, the robustness of the parameter estimates against the different sources of noise should be studied and analyzed. This is extremely important to especially assess arterial stiffness.

\section{CONCLUSION}

A deep understanding of the biomechanical properties of the vascular system afford an important insight into the mechanisms of arterial stiffening. The appearance of fractional-order behavior in the arterial system has been identified by many experimental studies of the viscoelasticity properties of the collagenous tissues in the arterial bed; the analysis of the arterial blood flow and red blood cell membrane mechanics, and the characterization of the heart valve cusp. In this paper, we introduced a fractional-order novel paradigm to estimate and assess the apparent vascular compliance. Accordingly, we have proposed five model representations. The proposed models present different structures that incorporate fractional-order capacitors (FOC) and ideal resistors and capacitors to display the dynamic relationship between the blood volume and aortic input pressure. FOC, which generalizes capacitors and resistors, displays a fractional-order behavior that captures both elastic and viscous properties through a power-law formulation.

The comparison between the proposed models and the standard apparent compliance models showed performance variations and a compromise between the number of parameters per representation (complexity) and accuracy. In fact, complicated configurations give better RMSE at the expense of the complexity. However, the results also demonstrate that model system fractionalizing is enhancing the accuracy of the arterial compliance while reducing the complexity. For instance, it has been found that a model with only one FOC in series with a resistor (referred to Model (C)) is able to perform an acceptable RMSE. Besides, in this work we explored the interpretability of the fractionalorder parameters by checking the correlation between the fractional differentiation-order, $\alpha$, and its associated hysteresivity coefficient, $\eta_{r}$, and the main hemodynamic determinants \{aortic systolic blood pressure (SBP), aortic diastolic blood pressure (DBP), aortic pulse pressure (APP), aortic pulse wave velocity $\left(P W V_{a}\right)$ and carotid-femoral pulse wave velocity $\left.\left(P W V_{c f}\right)\right\}$. The results showed good correlation coefficients of the fractional parameters and the central hemodynamic indices. The fractional-order-based approach of apparent arterial compliance has a great potential to provide a new alternative in assessing arterial stiffness.

Future investigations will be directed toward integrating the nonlinear elastic vessel properties in the evaluation of the apparent frequency-dependent apparent compliance. Also, it is vital to conduct in-vivo validation of the proposed approach. Finally, it is worth integrating the proposed model representation of the apparent vascular compliance within a complete lumped-parameter model for the whole systemic circulation and study the effects of certain cardiovascular diseases upon changes in the dynamic arterial compliance represented by the fractional-order capacitor.

To enable future authors to perform reproducible and easily-compared assessments of novel algorithms, we provide a publicly available Matlab code used for the numerical implementation and models calibration in this study. The preprocessed data investigated in this study is also available along with the statistical analysis tools. The algorithms and data are available at https://github.com/EMANGKAUST/Fractional-modeling-of-vascular-compliance We welcome developments to the existing code or contributions of new algorithms for inclusion in future versions of the arterial stiffness prediction platform.

\section{ACKNOWLEDGMENT}

Research reported in this publication was supported by King Abdullah University of Science and Technology (KAUST) Base Research Fund (BAS/1/162701-01). Additionally, the authors would like to thank Dr. Ali Haneef, associate consultant cardiac surgeon and co-chairman quality management at King Faisal Cardiac Center, King Abdulaziz Medical City, National Guard Health Affairs, in the Western Region, Jeddah, KSA and Dr. Nesrine T. Bahloul, medical intern at Department of Pediatrics, Sfax Medical School, Hedi Chaker Hospital, Sfax, Tunisia, for their assistance and valuable advices.

\section{REFERENCES}

[1] F. Liang and H. Liu, "A closed-loop lumped parameter computational model for human cardiovascular system," JSME International Journal Series C Mechanical Systems, Machine Elements and Manufacturing, vol. 48, no. 4, pp. 484-493, 2005. 
[2] T. Alderliesten, M. K. Konings, and W. J. Niessen, "Simulation of minimally invasive vascular interventions for training purposes," Computer Aided Surgery, vol. 9, no. 1-2, pp. 3-15, 2004.

[3] E. Van Disseldorp, N. Petterson, M. Rutten, F. Van De Vosse, M. van Sambeek, and R. Lopata, "Patient specific wall stress analysis and mechanical characterization of abdominal aortic aneurysms using $4 \mathrm{~d}$ ultrasound," European Journal of Vascular and Endovascular Surgery, vol. 52, no. 5, pp. 635-642, 2016.

[4] B. W. Beulen, N. Bijnens, G. G. Koutsouridis, P. J. Brands, M. C. Rutten, and F. N. van de Vosse, "Toward noninvasive blood pressure assessment in arteries by using ultrasound," Ultrasound in medicine \& biology, vol. 37, no. 5, pp. 788-797, 2011.

[5] W. Huberts, S. G. Heinen, N. Zonnebeld, D. A. van den Heuvel, J.P. P. de Vries, J. H. Tordoir, D. R. Hose, T. Delhaas, and F. N. van de Vosse, "What is needed to make cardiovascular models suitable for clinical decision support? a viewpoint paper," Journal of computational science, vol. 24, pp. 68-84, 2018.

[6] C. Leguy, E. Bosboom, H. Gelderblom, A. Hoeks, and F. Van De Vosse, "Estimation of distributed arterial mechanical properties using a wave propagation model in a reverse way," Medical engineering \& physics, vol. 32, no. 9, pp. 957-967, 2010.

[7] N. Stergiopulos, J. Meister, and N. Westerhof, "Evaluation of methods for estimation of total arterial compliance," American Journal of Physiology-Heart and Circulatory Physiology, vol. 268, no. 4, pp. H1540-H1548, 1995.

[8] N. Stergiopulos, B. E. Westerhof, and N. Westerhof, "Total arterial inertance as the fourth element of the windkessel model," American Journal of Physiology-Heart and Circulatory Physiology, vol. 276, no. 1, pp. H81-H88, 1999.

[9] I. Mackenzie, I. Wilkinson, and J. Cockcroft, "Assessment of arterial stiffness in clinical practice," Qjm, vol. 95, no. 2, pp. 67-74, 2002.

[10] B. Haluska, L. Jeffriess, J. Brown, S. Carlier, and T. Marwick, “A comparison of methods for assessing total arterial compliance," Journal of human hypertension, vol. 24, no. 4, pp. 254-262, 2010.

[11] D. Valdez-Jasso, D. Bia, Y. Zócalo, R. L. Armentano, M. A. Haider, and M. S. Olufsen, "Linear and nonlinear viscoelastic modeling of aorta and carotid pressure-area dynamics under in vivo and ex vivo conditions," Annals of biomedical engineering, vol. 39, no. 5, pp. 1438-1456, 2011.

[12] N. Stergiopulos, P. Segers, and N. Westerhof, "Use of pulse pressure method for estimating total arterial compliance in vivo," American Journal of Physiology-Heart and Circulatory Physiology, vol. 276, no. 2, pp. H424-H428, 1999.

[13] N. Westerhof, J.-W. Lankhaar, and B. E. Westerhof, "The arterial windkessel," Medical \& biological engineering \& computing, vol. 47, no. 2, pp. 131-141, 2009.

[14] P. Segers, P. Verdonck, Y. Deryck, S. Brimioulle, R. Naeije, S. Carlier, and N. Stergiopulos, "Pulse pressure method and the area method for the estimation of total arterial compliance in dogs: sensitivity to wave reflection intensity," Annals of biomedical engineering, vol. 27, no. 4, pp. 480-485, 1999.

[15] G. de Simone, M. J. Roman, M. J. Koren, G. A. Mensah, A. Ganau, and R. B. Devereux, "Stroke volume/pulse pressure ratio and cardiovascular risk in arterial hypertension," Hypertension, vol. 33, no. 3, pp. 800-805, 1999.

[16] D. Chemla, J.-L. Hébert, C. Coirault, K. Zamani, I. Suard, P. Colin, and Y. Lecarpentier, "Total arterial compliance estimated by stroke volume-to-aortic pulse pressure ratio in humans," American journal of physiology-Heart and circulatory physiology, vol. 274, no. 2, pp. H500-H505, 1998.

[17] M. Kaya, V. Balasubramanian, A. Patel, Y. Ge, and J. K. Li, "A novel compliance-pressure loop approach to quantify arterial compliance in systole and in diastole," Computers in biology and medicine, vol. 99, pp. 98-106, 2018.

[18] Y. Ge, "A new approach to assess arterial system function with compliance-pressure loop," Ph.D. dissertation, Rutgers UniversitySchool of Graduate Studies, 2018.

[19] C. M. Quick, D. S. Berger, and A. Noordergraaf, "Apparent arterial compliance," American Journal of Physiology-Heart and Circulatory Physiology, vol. 274, no. 4, pp. H1393-H1403, 1998.

[20] C. M. Quick, D. S. Berger, D. A. Hettrick, and A. Noordergraaf, "True arterial system compliance estimated from apparent arterial compliance," Annals of biomedical engineering, vol. 28, no. 3, pp. 291-301, 2000.

[21] D. Craiem and R. Armentano, "The new apparent compliance concept as a simple lumped model," Cardiovascular Engineering: An International Journal, vol. 3, no. 2, pp. 81-83, 2003.

[22] R. Burattini and S. Natalucci, "Complex and frequency-dependent compliance of viscoelastic windkessel resolves contradictions in elastic windkessels," Medical engineering \& physics, vol. 20, no. 7, pp. 502514, 1998.

[23] R. Visaria, Modeling of cardiovascular system, pulmonary mechanics and gas exchange. The University of Utah, 2005.

[24] C. M. Ionescu, J. T. Machado, and R. De Keyser, "Modeling of the lung impedance using a fractional-order ladder network with constant phase elements," IEEE Transactions on biomedical circuits and systems, vol. 5, no. 1, pp. 83-89, 2010.

[25] Y. Kobayashi, A. Kato, H. Watanabe, T. Hoshi, K. Kawamura, and M. G. Fujie, "Modeling of viscoelastic and nonlinear material properties of liver tissue using fractional calculations," Journal of Biomechanical Science and Engineering, vol. 7, no. 2, pp. 177-187, 2012.

[26] R. L. Magin, Fractional calculus in bioengineering. Begell House Redding, 2006.

[27] A. Jaishankar and G. H. McKinley, "Power-law rheology in the bulk and at the interface: quasi-properties and fractional constitutive equations," Proceedings of the Royal Society A: Mathematical, Physical and Engineering Sciences, vol. 469, no. 2149, p. 20120284, 2013.

[28] D. Craiem and R. L. Armentano, "A fractional derivative model to describe arterial viscoelasticity," Biorheology, vol. 44, no. 4, pp. 251263, 2007.

[29] D. Craiem, F. J. Rojo, J. M. Atienza, R. L. Armentano, and G. V. Guinea, "Fractional-order viscoelasticity applied to describe uniaxial stress relaxation of human arteries," Physics in Medicine \& Biology, vol. 53, no. 17 , p. 4543, 2008.

[30] D. Craiem and R. L. Magin, "Fractional order models of viscoelasticity as an alternative in the analysis of red blood cell (rbc) membrane mechanics," Physical biology, vol. 7, no. 1, p. 013001, 2010.

[31] P. Perdikaris and G. E. Karniadakis, "Fractional-order viscoelasticity in one-dimensional blood flow models," Annals of biomedical engineering, vol. 42, no. 5, pp. 1012-1023, 2014.

[32] J. P. Zerpa, A. Canelas, B. Sensale, D. B. Santana, and R. Armentano, "Modeling the arterial wall mechanics using a novel high-order viscoelastic fractional element," Applied Mathematical Modelling, vol. 39, no. 16, pp. 4767-4780, 2015.

[33] M. A. Bahloul and T. M. Laleg-Kirati, "Three-element fractionalorder viscoelastic arterial windkessel model," in 2018 40th Annual International Conference of the IEEE Engineering in Medicine and Biology Society (EMBC). IEEE, 2018, pp. 5261-5266.

[34] _ "Arterial viscoelastic model using lumped parameter circuit with fractional-order capacitor," in 2018 IEEE 61st International Midwest Symposium on Circuits and Systems (MWSCAS). IEEE, 2018, pp. 53-56.

[35] M. A. Bahloul and T.-M. L. Kirati, "Fractional order models of arterial windkessel as an alternative in the analysis of the left ventricular afterload," arXiv preprint arXiv:1908.05239, 2019.

[36] M. A. Bahloul and T.-M. Laleg-Kirati, "Assessment of fractional-order arterial windkessel as a model of aortic input impedance," IEEE Open Journal of Engineering in Medicine and Biology, vol. 1, pp. 123-132, 2020.

[37] M. A. Bahloul and T.-M. L. Kirati, "Two-element fractional-order windkessel model to assess the arterial input impedance," in 201941 st Annual International Conference of the IEEE Engineering in Medicine and Biology Society (EMBC). IEEE, 2019, pp. 5018-5023.

[38] M. Nakagawa and K. Sorimachi, "Basic characteristics of a fractance device," IEICE Transactions on Fundamentals of Electronics, Communications and Computer Sciences, vol. 75, no. 12, pp. 1814-1819, 1992.

[39] G. Tsirimokou, "A systematic procedure for deriving rc networks of fractional-order elements emulators using matlab," AEU-International Journal of Electronics and Communications, vol. 78, pp. 7-14, 2017.

[40] C. M. Ionescu, The human respiratory system: an analysis of the interplay between anatomy, structure, breathing and fractal dynamics. Springer Science \& Business Media, 2013. 
[41] T. C. Doehring, A. D. Freed, E. O. Carew, and I. Vesely, "Fractional order viscoelasticity of the aortic valve cusp: an alternative to quasilinear viscoelasticity," Journal of biomechanical engineering, vol. 127, no. 4 , pp. 700-708, 2005.

[42] P. Charlton, J. Mariscal Harana, S. Vennin, Y. Li, P. Chowienczyk, a database for in silico evaluation of haemodynamics and pulse wave indices," American Journal of Physiology-Heart and Circulatory Physiology, 2019.

[43] J. Alastruey, K. H. Parker, S. J. Sherwin et al., "Arterial pulse wave haemodynamics," in 11th International Conference on Pressure Surges, vol. 30. Virtual PiE Led t/a BHR Group Lisbon, Portugal, 2012, pp. 401-443.

[44] P. H. Charlton, P. Celka, B. Farukh, P. Chowienczyk, and J. Alastruey, "Assessing mental stress from the photoplethysmogram: a numerical study," Physiological measurement, vol. 39, no. 5, p. 054001, 2018.

[45] G. R. Boss and J. E. Seegmiller, "Age-related physiological changes and their clinical significance," Western Journal of Medicine, vol. 135, no. 6 , p. 434, 1981.

[46] B. Folkow and A. Svanborg, "Physiology of cardiovascular aging," Physiological reviews, vol. 73, no. 4, pp. 725-764, 1993.

[47] A. Kawamoto, K. Shimada, K. Matsubayashi, T. Chikamori, O. Kuzume, H. Ogura, and T. Ozawa, "Cardiovascular regulatory functions in elderly patients with hypertension." Hypertension, vol. 13, no. 5 , pp. 401-407, 1989.

[48] E. G. Lakatta, "Cardiovascular regulatory mechanisms in advanced age." Physiological reviews, vol. 73, no. 2, pp. 413-467, 1993.

[49] _ - "Age-associated cardiovascular changes in health: impact on cardiovascular disease in older persons," Heart failure reviews, vol. 7,

[50] E. G. Lakatta, J. H. Mitchell, A. Pomerance, and G. G. Rowe, "Human aging: changes in structure and function," Journal of the American College of Cardiology, vol. 10, no. 2S1, pp. 42A-47A, 1987.

[51] C. M. McEniery, I. B. Wilkinson, and A. P. Avolio, "Age, hypertension and arterial function," Clinical and Experimental Pharmacology and Physiology, vol. 34, no. 7, pp. 665-671, 2007.

[52] M. F. O'Rourke, "Arterial aging: pathophysiological principles," Vascular medicine, vol. 12, no. 4, pp. 329-341, 2007.

[53] M. F. O'rourke and J. Hashimoto, "Mechanical factors in arterial aging a clinical perspective," Journal of the American College of Cardiology, vol. 50, no. 1, pp. 1-13, 2007.

[54] M. Safar, "Ageing and its effects on the cardiovascular system," Drugs,

[55] P. H. Charlton, J. Mariscal Harana, S. Vennin, M. Willemet, P. Chowienczyk, and J. Alastruey, "Modelling arterial pulse wave propagation during healthy ageing," in 8th World Congress of Biomechanic, 2018, pp. 8-12.

[56] A. Coccarelli, H. M. Hasan, J. Carson, D. Parthimos, and P. Nithiarasu, "Influence of ageing on human body blood flow and heat transfer: a detailed computational modelling study," International journal for numerical methods in biomedical engineering, vol. 34 , no. 10, p. e3120, 2018.

[57] A. Guala, C. Camporeale, and L. Ridolfi, "Compensatory effect between aortic stiffening and remodelling during ageing," PloS one, vol. 10, no. 10, p. e0139211, 2015.

[58] A. Guala, C. Camporeale, L. Ridolfi, and L. Mesin, "Non-invasive aortic systolic pressure and pulse wave velocity estimation in a primary care setting: an in silico study," Medical engineering \& physics, vol. 42, pp. 91-98, 2017.

[59] E. Maksuti, N. Westerhof, B. E. Westerhof, M. Broomé, and N. Stergiopulos, "Contribution of the arterial system and the heart to blood pressure during normal aging-a simulation study," PloS one, vol. 11, no. 6 , p. e0157493, 2016

[60] S. Pagoulatou and N. Stergiopulos, "Evolution of aortic pressure during normal ageing: A model-based study," PLoS One, vol. 12, no. 7, p. e0182173, 2017.

[61] A. D. Pontoriero, P. H. Charlton, and J. Alastruey, "Alzheimer's disease: a step towards prognosis using smart wearables," in Multidisciplinary Digital Publishing Institute Proceedings, vol. 4, no. 1, 2018, p. 8.

[62] J. P. Mynard and J. J. Smolich, "One-dimensional haemodynamic modeling and wave dynamics in the entire adult circulation," Annals of biomedical engineering, vol. 43, no. 6, pp. 1443-1460, 2015. and J. Alastruey, "Modelling arterial pulse waves in healthy ageing:

[63] M. Willemet, P. Chowienczyk, and J. Alastruey, "A database of virtual healthy subjects to assess the accuracy of foot-to-foot pulse wave velocities for estimation of aortic stiffness," American Journal of Physiology-Heart and Circulatory Physiology, vol. 309, no. 4, pp. H663-H675, 2015.

[64] W. Goedhard and A. Knoop, "A model of the arterial wall," Journal of biomechanics, vol. 6, no. 3, pp. 281-288, 1973.

[65] D. Craiem, F. Rojo, J. Atienza, G. Guinea, and R. L. Armentano, "Fractional calculus applied to model arterial viscoelasticity," Latin American applied research, vol. 38, no. 2, pp. 141-145, 2008. no. 1, pp. 29-49, 2002. vol. 39 , no. 1 , pp. $1-8,1990$.
[66] I. Podlubny, Fractional differential equations: an introduction to fractional derivatives, fractional differential equations, to methods of their solution and some of their applications. Elsevier, 1998, vol. 198.

[67] G. Jumarie, "Modified riemann-liouville derivative and fractional taylor series of nondifferentiable functions further results," Computers \& Mathematics with Applications, vol. 51, no. 9-10, pp. 1367-1376, 2006.

\section{APPENDIX A}

\section{APPARENT VASCULAR COMPLIANCE}

Based on the conservation mass, the flow into the arterial tree $\left(Q_{i n}\right)$ is equal to the flow stored $\left(Q_{\text {stored }}\right)$ plus the flow out of the arterial tree $\left(Q_{\text {out }}\right)$.

$$
Q_{\text {in }}=Q_{\text {stored }}+Q_{\text {out }} \text {. }
$$

$Q_{\text {out }}$ can be expressed as:

$$
Q_{\text {out }}(w)=\frac{1}{R_{\text {app }}(\omega)} P_{\omega}(w),
$$

where $\omega$ corresponds to the angular frequency and $R_{a p p}$ is the apparent arterial resistance [19].

$Q_{\text {stored }}$ is defined as the rate of flow by taking the first derivative of the blood volume $(V)$ equation for the time.

$$
Q_{\text {stored }}(t)=\frac{d V}{d t}=\frac{d V(t)}{d P_{\text {in }}(t)} \frac{d P_{\text {in }}(t)}{d t},
$$

The apparent vascular compliance, $\left(C_{a p p}\right)$, is defined as the derivative of stored volume with respect to the input pressure pressure $\left(P_{\text {in }}\right)$.

$$
C_{\text {app }}=\frac{V(\omega)}{P_{\text {in }}(\omega)}
$$

Hence $Q_{\text {stored }}$ can be expressed as:

$$
Q_{\text {stored }}=j \omega P_{\text {in }} C_{a p p} .
$$

The aortic input impedance $Z_{\text {in }}$ describes the ability of the arterial system to hamper the blood flow dynamically. Functionally, $Z_{i n}$ is defined as the dynamic relationship, in the frequency domain, of the arterial blood pressure $\left(P_{i n}\right)$ and blood flow $\left(Q_{i n}\right)$ at the entrance of the systemic circulatory system, that is:

$$
Z_{\text {in }}(\omega)=\frac{P_{\text {in }}(\omega)}{Q_{\text {in }}(\omega)} .
$$

Substituting equations (A2) and (A5) into equation (A1) gives:

$$
Q_{i n}=j \omega P_{i n} C_{a p p}+\frac{1}{R_{a p p}(\omega)} P_{\omega}(w) .
$$


Rearranging (A7) yields an expression for $C_{a p p}$ in terms of Zin and $R_{a p p}$ as follow:

$$
C_{a p p}=\frac{R_{a p p}-Z_{i n}}{j \omega R_{a p p} Z_{i n}} .
$$

\section{APPENDIX B \\ FRACTIONAL CALCULUS}

Over recent decades, the theory of fractional calculus has gained a significant research interest in the field of biology [26]. This is originated from the interdisciplinary nature of this field as well as the flexibility and effectiveness of FC in describing complicated physical systems. For example, the characterization of bio-impedance, modeling of the viscoelasticity and biological cells, and representing the mechanical properties of the arterial system, as well as respiratory systems, have been investigated extensively through the exploring of FC. The concept of FC is not new dating from the pioneer conversation between L'Hopital and Leibniz in 1695 that yielded to the generalization of the conventional integer derivative to a non-integer order operator [66], as follow:

$$
D_{t}^{\alpha}=\left\{\begin{array}{ccc}
\frac{\mathrm{d}^{\alpha}}{\mathrm{d} t^{\alpha}} & \text { if } & \alpha>0 \\
1, & \text { if } & \alpha=0 \\
\int_{t}^{0}(d f)^{-\alpha} & \text { if } & \alpha<0
\end{array}\right.
$$

where $\alpha \in \mathbb{R}$ is the order of the operator known as the fractional-order, and $d f$ is the derivative function. Numerous fractional calculus definitions have been suggested. Generally, these definitions can be classified into two main classes. In the first class, the operator $D_{t}^{\alpha}$ is converted into the standard differential-integral operator when $\alpha$ is integer. For instance, according to the Reimann-Liouville definition, the fractional-order derivative $\alpha$ of a function $g(t)$ can be formulated [67] as follow:

$$
D_{t}^{\alpha} g(t)=\frac{1}{\Gamma(1-\alpha)} \frac{\mathrm{d}}{\mathrm{d} t} \int_{0}^{t} \frac{g(\tau)}{(1-\tau)^{\alpha}} d \tau,
$$

where $\Gamma$ is the Euler gamma function. The second class is that the Laplace transform of $D_{t}^{\alpha}$ is $s^{\alpha}$, assuming a null initial fractional conditions. The fractional operator is given by:

$$
D_{t}^{\alpha} g(t) \stackrel{L}{\rightarrow} s^{\alpha} G(s)
$$

This class is very interesting in developing parametric models for complex systems and control design in frequency domain. where $s$ is the complex Laplace. The Fourier transform can be found by substituting $s$ by $j w$ and thus the equivalent frequency-domain expression of $s^{n}$ are:

$$
\begin{aligned}
& (j \omega)^{\alpha}=\omega^{\alpha}\left(\cos \frac{\alpha \pi}{2}-j \sin \frac{\alpha \pi}{2}\right), \\
& \frac{1}{(j \omega)^{\alpha}}=\frac{1}{\omega^{\alpha}}\left(\cos \frac{\alpha \pi}{2}+j \sin \frac{\alpha \pi}{2}\right) .
\end{aligned}
$$




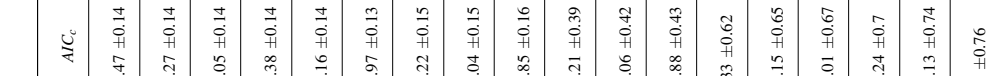

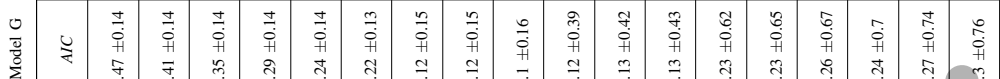

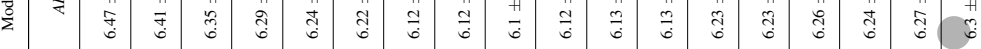

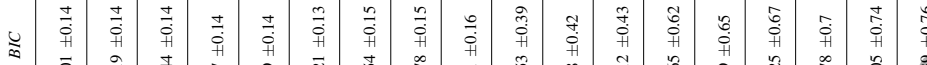



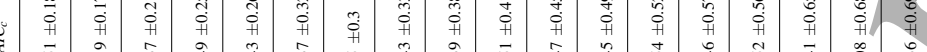

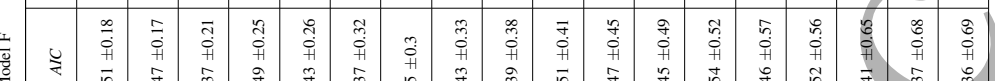

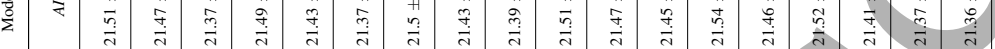

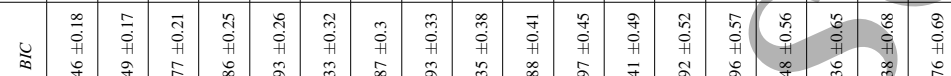

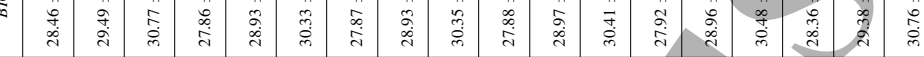

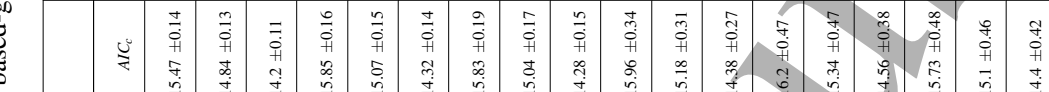

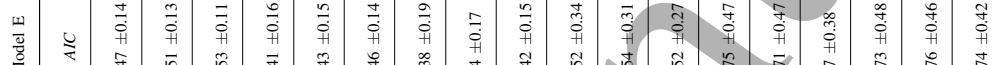
噌

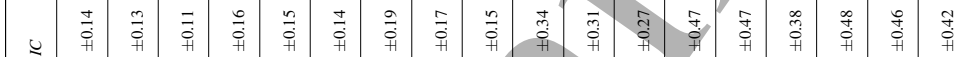

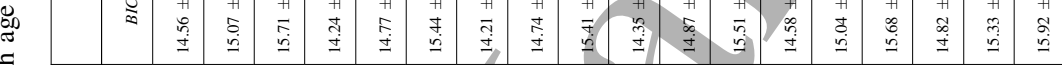
ల్ల

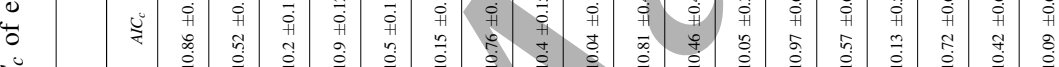
$\stackrel{7}{\longleftarrow}$

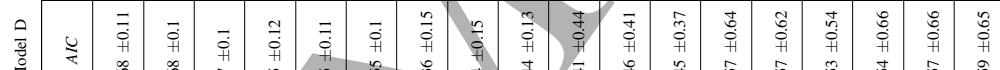

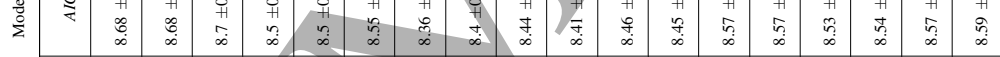





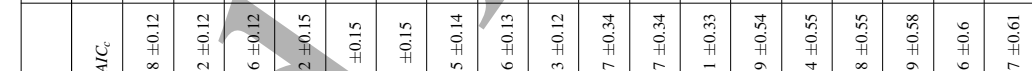

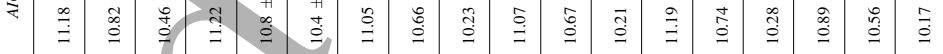

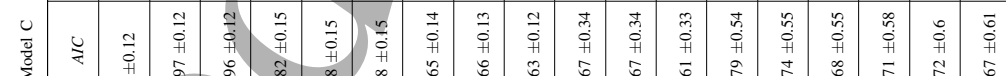

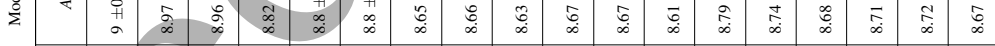

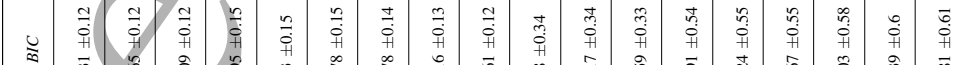

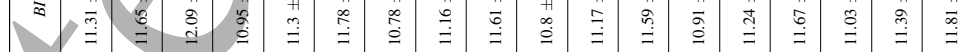

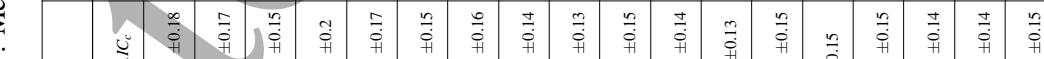
Z ₹

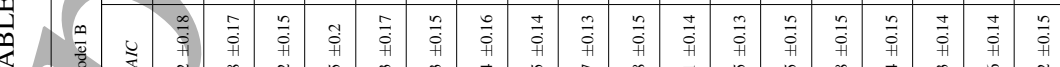

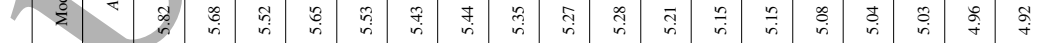

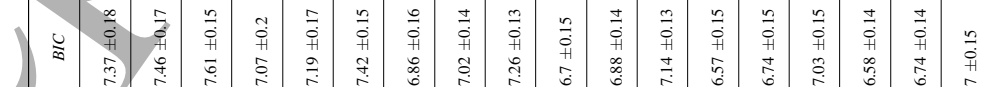

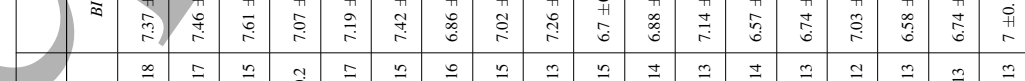

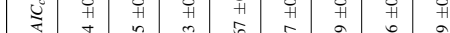

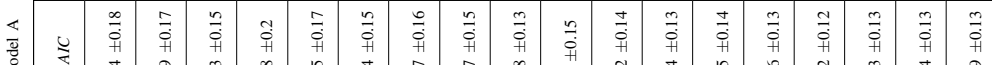

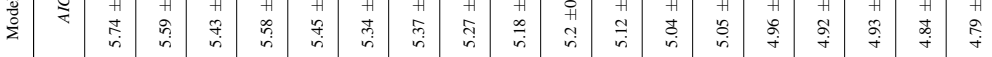

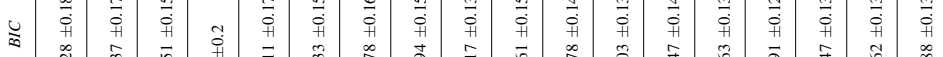



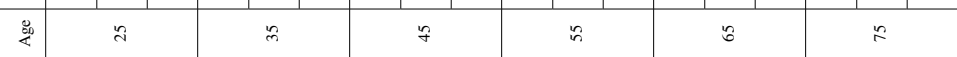







